\title{
On the Yamabe problem and the scalar curvature problems under boundary conditions
}

\author{
Antonio Ambrosetti · YanYan Li • Andrea Malchiodi \\ Received: 18 May 2000 / \\ Published online: 28 February 2002 - (C) Springer-Verlag 2002
}

\section{Introduction}

In this paper we prove some existence results concerning a problem arising in conformal differential geometry. Consider a smooth metric $g$ on $B=\left\{x \in \mathbb{R}^{n}\right.$ : $|x|<1\}$, the unit ball on $\mathbb{R}^{n}, n \geq 3$, and let $\Delta_{g}, R_{g}, v_{g}, h_{g}$ denote, respectively, the Laplace-Beltrami operator, the scalar curvature of $(B, g)$, the outward unit normal to $\partial B=S^{n-1}$ with respect to $g$ and the mean curvature of $\left(S^{n-1}, g\right)$. Given two smooth functions $R^{\prime}$ and $h^{\prime}$, we will be concerned with the existence of positive solutions $u \in H^{1}(B)$ of

$$
\left\{\begin{array}{l}
-4 \frac{(n-1)}{(n-2)} \Delta_{g} u+R_{g} u=R^{\prime} u^{\frac{n+2}{n-2}}, \text { in } B \\
\frac{2}{(n-2)} \partial_{v_{g}} u+h_{g} u=h^{\prime} u^{\frac{n}{n-2}}, \text { on } \partial B=S^{n-1} .
\end{array}\right.
$$

It is well known that such a solution is $C^{\infty}$ provided $g, R^{\prime}$ and $h^{\prime}$ are, see [10]. If $u>0$ is a smooth solution of (1) then $g^{\prime}=u^{4 /(n-2)} g$ is a metric, conformally equivalent to $g$, such that $R^{\prime}$ and $h^{\prime}$ are, respectively, the scalar curvature of $\left(B, g^{\prime}\right)$ and the mean curvature of $\left(S^{n-1}, g^{\prime}\right)$. Up to a stereographic projection, this is equivalent to finding a conformal metric on the upper half sphere $S_{+}^{n}=\left\{\left(x_{1}, \ldots, x_{n+1}\right) \in \mathbb{R}^{n+1}:|x|=1, x_{n+1}>0\right\}$ such that the scalar curvature of $S_{+}^{n}$ and the mean curvature of $\partial S_{+}^{n}=S^{n-1}$ are prescribed functions.

\section{A. Ambrosetti}

S.I.S.S.A., via Beirut, 2-4, 34019 Trieste, Italy (e-mail: ambr@ sissa.it)

Y.Y. LI, A. MALChIODI

Department of Mathematics, Rutgers University New Brunswick, NJ 08903, USA

(e-mail: yyli@math.rutgers.edu; malchiod@math.rutgers.edu)

The first and the third authors have been supported by the italian M.U.R.S.T. under the national project Variational Methods and Nonlinear Differential Equations. 
In the first part of the paper we deal with the the case in which $R^{\prime}$ and $h^{\prime}$ are constant, say $R^{\prime} \equiv 1$ and $h^{\prime} \equiv c$, when (1) becomes

$$
\left\{\begin{array}{l}
-4 \frac{(n-1)}{(n-2)} \Delta_{g} u+R_{g} u=u^{\frac{n+2}{n-2}}, \text { in } B ; \\
\frac{2}{(n-2)} \partial_{\nu_{g}} u+h_{g} u=c u^{\frac{n}{n-2}}, \text { on } \partial B=S^{n-1} .
\end{array}\right.
$$

This will be referred as the Yamabe like problem and was first studied in [10$12]$. More recently, the existence of a solution of (1) has been proved in $[14,15]$ under the assumption that $(B, g)$ is of positive type (for a definition see [14]) and satisfies one of the following assumptions:

(i) $(B, g)$ is locally conformally flat and $\partial B$ is umbilical;

(ii) $n \geq 5$ and $\partial B$ is not umbilical.

Our main result concerning the Yamabe like problem shows that none of $(i)$ or (ii) is required when $g$ is close to the standard metric $g_{0}$ on $B$. Precisely, consider the following class $\mathcal{G}_{\varepsilon}$ of bilinear forms

$$
\mathcal{G}_{\varepsilon}=\left\{g \in C^{\infty}(B):\left\|g-g_{0}\right\|_{L^{\infty}(B)} \leq \varepsilon,\|\nabla g\|_{L^{n}(B)} \leq \varepsilon,\|\nabla g\|_{L^{n-1}\left(S^{n-1}\right)} \leq \varepsilon\right\} .
$$

Inequalities in (2) hold if for example $\left\|g-g_{0}\right\|_{C^{1}(B)} \leq \varepsilon$, or if $\left\|g-g_{0}\right\|_{W^{2, n}(B)} \leq \varepsilon$. We will show:

Theorem 1. Given $M>0$ there exists $\varepsilon_{0}>0$ such that for every $\varepsilon$ with $\varepsilon \in$ $\left(0, \varepsilon_{0}\right)$, for every $c>-M$ and for every metric $g \in \mathcal{G}_{\varepsilon}$ problem $(Y)$ possesses a positive solution.

In the second part of the paper we will take $g=g_{0}, R^{\prime}=1+\varepsilon K(x)$, $h^{\prime}=c+\varepsilon h(x)$ and consider the Scalar Curvature like problem

$$
\left\{\begin{array}{l}
-4 \frac{(n-1)}{(n-2)} \Delta u=(1+\varepsilon K(x)) u^{\frac{n+2}{n-2}}, \text { in } B \\
\frac{2}{(n-2)} \frac{\partial u}{\partial v}+u=(c+\varepsilon h(x)) u^{\frac{n}{n-2}}, \text { on } S^{n-1},
\end{array}\right.
$$

where $v=v_{g_{0}}$. The Scalar Curvature like problem has been studied in [16] where a non perturbative problem like

$$
\left\{\begin{array}{l}
-4 \frac{(n-1)}{(n-2)} \Delta u=R^{\prime}(x) u^{\frac{n+2}{n-2}}, \text { in } B \\
\frac{2}{(n-2)} \frac{\partial u}{\partial v}+u=0, \text { on } S^{n-1},
\end{array}\right.
$$


has been considered. We also mention the paper [9] dealing with the existence of solutions of

$$
\left\{\begin{array}{l}
\Delta u=0, \text { in } B \\
\frac{2}{(n-2)} \frac{\partial u}{\partial v}+u=(1+\varepsilon h(x)) u^{\frac{n}{n-2}}, \text { on } S^{n-1},
\end{array}\right.
$$

a problem similar in nature to $\left(P_{\varepsilon}\right)$.

To give an idea of the existence results we can prove, let us consider the particular cases that either $h \equiv 0$ or $K \equiv 0$. In the former, problem $\left(P_{\varepsilon}\right)$ becomes

$$
\left\{\begin{array}{l}
-4 \frac{(n-1)}{(n-2)} \Delta u=(1+\varepsilon K(x)) u^{\frac{n+2}{n-2}}, \text { in } B \\
\frac{2}{(n-2)} \frac{\partial u}{\partial v}+u=c u^{\frac{n}{n-2}}, \text { on } S^{n-1},
\end{array}\right.
$$

Theorem 2. Suppose that $K$ satisfies

$\left(K_{1}\right)$ there exists an absolute maximum (resp. minimum) $p$ of $\left.K\right|_{S^{n-1}}$ such that $K^{\prime}(p) \cdot p<0$, resp. $K^{\prime}(p) \cdot p>0$.

Then for $|\varepsilon|$ sufficiently small, $\left(P_{\varepsilon, K}\right)$ has a positive solution.

Another kind of result is the following

Theorem 3. Let $\left.K\right|_{S^{n-1}}$ be a Morse function and satisfies

$$
\begin{gathered}
K^{\prime}(x) \cdot x \neq 0, \quad \forall x \in \operatorname{Crit}\left(\left.K\right|_{S^{n-1}}\right) \\
\sum_{x \in \operatorname{Crit}\left(\left.K\right|_{\left.S^{n-1}\right): K^{\prime}(x) \cdot x<0}\right.}(-1)^{m(x, K)} \neq 1,
\end{gathered}
$$

where $m(x, K)$ is the Morse index of $\left.K\right|_{S^{n-1}}$ at $x$. Then for $|\varepsilon|$ sufficiently small, problem $\left(P_{\varepsilon, K}\right)$ has a positive solution.

When $K \equiv 0$ problem $\left(P_{\varepsilon}\right)$ becomes

$$
\begin{cases}-4 \frac{(n-1)}{(n-2)} \Delta u=u^{\frac{n+2}{n-2}}, & \text { in } B \\ \frac{2}{(n-2)} \frac{\partial u}{\partial v}+u=(c+\varepsilon h(x)) u^{\frac{n}{n-2}}, & \text { on } S^{n-1}\end{cases}
$$


Theorem 4. Let $h \in C^{\infty}\left(S^{n-1}\right)$ be a Morse function satisfying:

$$
\begin{gathered}
\Delta_{T} h(x) \neq 0, \quad \forall x \in \operatorname{Crit}(h) ; \\
\sum_{x \in \operatorname{Crit}(h): \Delta_{T} h(x)<0}(-1)^{m(x, h)} \neq 1,
\end{gathered}
$$

Then for $|\varepsilon|$ sufficiently small, problem $\left(P_{\varepsilon, h}\right)$ has a positive solution.

The preceding results are particular cases of more general ones, dealing with problem $\left(P_{\varepsilon}\right)$, where assumptions on a suitable combination of $K$ and $h$ are made. See Theorems 6 and 7 later on. For a comparison with the results of [9, 16], we refer to Remarks 5 and 6 in Sect. 4.

Solutions of the preceding problems are critical points of the energy functional $I^{c}=I_{g}^{c}: H^{1}(B) \rightarrow \mathbb{R}$,

$$
\begin{aligned}
I^{c}(u) & =2 \frac{(n-1)}{(n-2)} \int_{B}\left|\nabla_{g} u\right|^{2} d V_{g}+\frac{1}{2} \int_{B} R_{g} u^{2} d V_{g}-\frac{1}{2^{*}} \int_{B} R^{\prime} u^{2^{*}} d V_{g} \\
& +(n-1) \int_{\partial B} h_{g} u^{2} d \sigma_{g}-c(n-2) \int_{\partial B} h^{\prime}|u|^{2 \frac{n-1}{n-2}} d \sigma_{g} .
\end{aligned}
$$

In all the cases we will deal with, $I^{c}$ can be written in the form $I^{c}(u)=I_{0}^{c}(u)+$ $O(\varepsilon)$, where

$$
\begin{aligned}
I_{0}^{c}(u) & =2 \frac{(n-1)}{(n-2)} \int_{B}|\nabla u|^{2} d x+(n-1) \\
& \times \int_{\partial B} u^{2} d \sigma-\frac{1}{2^{*}} \int_{B}|u|^{2^{*}} d x-c(n-2) \int_{S^{n-1}}|u|^{2 \frac{n-1}{n-2}} d \sigma
\end{aligned}
$$

and can be faced by means of a perturbation method in critial point theory discussed in [1]. First, in Sect. 2, we show that $I_{0}^{c}$ has a finite dimensional manifold $Z^{c} \simeq B$ of critical points that is non degenerate, in the sense of [1], see Lemma 3. This allows us to perform a finite dimensional reduction (uniformly with respect to $c \geq-M$ ) that leads to seeking the critical points of $I^{c}$ constrained to $Z^{c}$. The proof of Theorem 1 is carried out in Sect. 3 and is mainly based upon the study of $I_{\mid Z^{c}}^{c}$. The lack of compactness inherited by $I^{c}$ is reflected on the fact that $Z^{c}$ is not closed. This difficulty is overcome using arguments similar to those emploied in $[3,7]$ : we show that $I^{c}$ can be extended to the boundary $\partial Z^{c}$ and there results $I_{\mid \partial Z^{c}}^{c} \equiv$ const., see Proposition 2.

In Sect. 4 we deal with the Scalar Curvature like problem. In this case there results $I^{c}(u)=I_{0}^{c}(u)+\varepsilon G(u)$, where $G$ depends upon $K$ and $h$ only, and one is lead to study the finite dimensional auxiliary functional $\Gamma=G_{\mid Z^{c}}$. More precisely, following the approach of [2], we evaluate $\Gamma$ on $\partial Z^{c}$, together with its first and second derivative. This permits to prove some general existence results which contain as particular cases Theorems 2, 3 and 4. The last part of Sect. 4 is 
devoted to a short discussion of the case in which $K, h$ inherit a simmetry. For example, if $K$ and $h$ are even functions, $\left(P_{\varepsilon}\right)$ has always a solution provided $\varepsilon$ is small, without any further assumption, see Theorem 8 .

Finally, in the Appendix we prove some technical Lemmas.

The main results of this paper has been annouced in [5].

\section{Notation}

$B$ denotes the unit ball in $\mathbb{R}^{n}$, centered at $x=0$.

We will work mainly in the functional space $H^{1}(B)$. In some cases it will be convenient to equip $H^{1}(B)$ with the scalar product

$$
(u, v)_{1}=4 \frac{(n-1)}{(n-2)} \int_{B} \nabla u \cdot \nabla v d x+2(n-1) \int_{\partial B} u v d \sigma
$$

that gives rise to the norm $\|u\|_{1}^{2}=(u, u)_{1}$, equivalent to the standard one.

If $E$ is an Hilbert space and $f \in C^{2}(E, \mathbb{R})$ is a functional, we denote by $f^{\prime}$ or $\nabla f$ its gradient; $f^{\prime \prime}(u): E \rightarrow E$ is the linear operator defined by duality in the following way

$$
\left(f^{\prime \prime}(u) v, w\right)=D^{2} f(u)[v, w], \quad \forall v, w \in E .
$$

$\sigma_{S}$ denotes the stereographic projection $\sigma_{S}: S^{n}=\left\{x \in \mathbb{R}^{n+1}|| x \mid=1\right\} \rightarrow$ $\mathbb{R}^{n}$ trough the south pole, where we identify $\mathbb{R}^{n}$ with $\left\{x \in \mathbb{R}^{n+1} \mid x_{n+1}=0\right\}$.

More in general, given $p \in S^{n}$, we denote by $\sigma_{p}: \mathbb{R}^{n} \rightarrow S^{n}$ the stereographic projection trough the point $p$.

The stereographic projections give rise to some isometries in the following way. The projection trough the south pole $S$ of $S^{n}$ gives rise to the isometry $\tau_{S}: H^{1}\left(S^{n}\right) \rightarrow H^{1}(B)$

$$
\tau_{S} u(x)=\frac{2}{1+|x|^{2}} u\left(\sigma_{S}^{-1} x\right), \quad x \in B
$$

Moreover, given $p \in \partial S_{+}^{n}$, the stereographic projection trough $p$ gives rise to the isometry $\tau_{p}: H^{1}\left(S_{+}^{n}\right) \rightarrow E=\mathcal{D}^{1,2}\left(\mathbb{R}_{+}^{n}\right)$ given by

$$
\tau_{p} u(x)=\frac{2}{1+|x|^{2}} u\left(\sigma_{p}^{-1} x\right), \quad x \in \mathbb{R}_{+}^{n} .
$$




\section{The unperturbed problem}

When $\varepsilon=0$, resp. $g=g_{0}$, problem $\left(P_{\varepsilon}\right)$, resp. $(Y)$, coincides with the unperturbed problem

$$
\begin{cases}-4 \frac{(n-1)}{(n-2)} \Delta u=u^{\frac{n+2}{n-2}}, & \text { in } B \\ \frac{2}{(n-2)} \partial_{\nu} u+u=c u^{\frac{n}{n-2}}, & \text { on } \partial B=S^{n-1} .\end{cases}
$$

Solutions of problem $(U P)$ can be found as critical points of the functional $I_{0}^{c}: H^{1}(B) \rightarrow \mathbb{R}$ defined as

$$
I_{0}^{c}(u)=\frac{1}{2}\|u\|_{1}^{2}-\frac{1}{2^{*}} \int_{B}|u|^{2^{*}} d x-c(n-2) \int_{S^{n-1}}|u|^{2 \frac{n-1}{n-2}} d \sigma .
$$

Consider the function $z_{0}: \mathbb{R}^{n} \rightarrow \mathbb{R}$,

$$
z_{0}(x)=\left(\frac{\kappa}{1+|x|^{2}}\right)^{\frac{n-2}{2}} ; \quad \kappa=\kappa_{n}=(4 n(n-1))^{\frac{1}{2}} .
$$

The function $z_{0}$ is the unique solution (up to translation and dilation) to the problem in $\mathbb{R}^{n}$

$$
-4 \frac{(n-1)}{(n-2)} \Delta u=u^{\frac{n+2}{n-2}}, \quad \text { in } \mathbb{R}^{n} ; \quad u>0 .
$$

We also set

$$
z_{\mu, \xi}=\mu^{-\frac{n-2}{2}} z_{0}((x-\xi) / \mu), \quad z_{\mu}=\mu^{-\frac{n-2}{2}} z_{0}(x / \mu) .
$$

By a stright calculation it follows that $z_{\mu, \xi}$ is a critical points of $I_{0}^{c}$, namely solutions of the problem $(U P)$, iff

$$
\mu^{2}+|\xi|^{2}-c \kappa \mu-1=0, \quad \mu>0 .
$$

The set

$$
Z^{c}=\left\{z_{\mu, \xi}: \mu^{2}+|\xi|^{2}-c \kappa \mu-1=0\right\}
$$

is an $n$-dimensional manifold, diffeomorphic to a ball in $\mathbb{R}^{n}$, with boundary $\partial Z^{c}$ corresponding to the parameter values $\mu=0,|\xi|=1$. 
We need to study the eigenvalues of $I_{0}^{\prime \prime}\left(z_{\mu, \xi}\right)$, with $z_{\mu, \xi} \in Z^{c}$. Recall that, by definition, $\lambda \in \mathbb{R}$ is an eigenvalue of $I_{0}^{\prime \prime}\left(z_{\mu, \xi}\right)$ if there exists $v \in H^{1}(B), v \neq 0$ such that $I_{0}^{\prime \prime}\left(z_{\mu, \xi}\right)[v]=\lambda v$ and this means that $v$ is solution of the linear problem

$$
\begin{cases}-4 \frac{(n-1)}{(n-2)}(1-\lambda) \Delta v=\frac{n+2}{n-2} z_{\mu, \xi}^{\frac{4}{n-2}} v, & \text { in } B \\ 4 \frac{(n-1)}{(n-2)}(1-\lambda) \partial_{\nu} v=2(n-1)\left(c \frac{n}{(n-2)} z_{\mu, \xi}^{\frac{2}{n-2}}+\lambda-1\right) v, & \text { on } S^{n-1} .\end{cases}
$$

The following lemma is well known.

Lemma 1. (a) $\lambda=0$ is an eigenvalue of (7) and the corresponding eigenspace is $n$ dimensional and coincides with the tangent space to $Z^{c}$ at $z_{\mu, \xi}$, namely is spanned by $D z_{\mu, \xi}$.

(b) (7) has precisely one negative eigenvalue $\lambda_{1}(c)$; all the remaining eigenvalues are positive.

Item $(a)$ is proved in [14]. Item $(b)$ easily follows from the fact that $z_{\mu, \xi}$ is a Mountain Pass critical point of $I_{0}^{c}$.

Let $\lambda_{2}(c)$ denote the smallest positive eigenvalue of $I_{0}^{\prime \prime}\left(z_{\mu, \xi}\right)$.

The main result of this section is the following one:

Lemma 2. For all $M>0$ there exists a positive constant $C_{M}$ such that

$$
\frac{1}{C_{M}} \leq\left|\lambda_{i}(c)\right| \leq C_{M}, \quad \forall c \geq-M, \quad i=1,2 .
$$

Remark. There is a numerical evidence that $\lambda_{2}(c) \downarrow 0$ as $c \downarrow-\infty$.

Proof. We will prove separately that $\left|\lambda_{i}(c)\right| \leq C_{M}$ and that $\frac{1}{C_{M}} \leq\left|\lambda_{i}(c)\right|$. For symmetry reasons it is sufficient to take $z_{\mu, \xi}=z_{\mu}$, namely to take $\xi=0$. In such a case $\mu$ depends only on $\xi$ and (5) yields

$$
\mu(c)=\frac{1}{2}\left(\kappa c+\sqrt{\kappa^{2} c^{2}+4}\right) .
$$

Case 1. $\left|\lambda_{i}(c)\right| \leq C_{M}$. By contradiction suppose there exists a sequence $c_{j} \rightarrow$ $+\infty$ such that $\left|\lambda_{i}\left(c_{j}\right)\right| \rightarrow+\infty, i=1,2$. Let $v_{j}$ denote an eigenfunction of (7) with $\lambda=\lambda_{i}\left(c_{j}\right)$. Then $v_{j}$ solves the problem

$$
\begin{cases}\Delta v_{j}=a_{j}(x) v_{j}, & \text { in } B \\ \partial_{\nu} v_{j}=b_{j}(x) v_{j}, & \text { on } S^{n-1},\end{cases}
$$


where

$$
\begin{aligned}
& a_{j}(x)=\frac{1}{\left(\lambda_{i}\left(c_{j}\right)-1\right)} \frac{n+2}{4(n-1)} z_{\mu\left(c_{j}\right)}^{\frac{4}{n-2}}(x), \quad x \in B \\
& b_{j}(x)=\frac{n-2}{2\left(1-\lambda_{i}\left(c_{j}\right)\right)}\left(c_{j} \frac{n}{(n-2)} z_{\mu\left(c_{j}\right)}^{\frac{2}{n-2}}(x)+\lambda_{i}\left(c_{j}\right)-1\right), \quad x \in S^{n-1} .
\end{aligned}
$$

Above, it is worth pointing out that $b_{j}$ is constant on $S^{n-1}$. Actually, there results

$$
z_{\mu}^{\frac{2}{n-2}}(x)=\kappa \mu^{-1}\left(1+\frac{1}{\mu^{2}}\right)^{-1}, \quad \forall x \in S^{n-1},
$$

and hence

$$
\begin{gathered}
b_{j} \equiv \frac{n-2}{2\left(1-\lambda_{i}\left(c_{j}\right)\right)}\left(c_{j} \frac{n}{(n-2)} \cdot \kappa \mu^{-1}\left(c_{j}\right)\left(1+\frac{1}{\mu^{2}\left(c_{j}\right)}\right)^{-1}+\lambda_{i}\left(c_{j}\right)-1\right), \\
\forall x \in S^{n-1} .
\end{gathered}
$$

Moreover, since $\mu \sim \kappa c$ as $c \rightarrow+\infty$, it turns out that

$$
b_{j} \rightarrow-\frac{(n-2)}{2} .
$$

Now, integrating by parts we deduce from (8)

$$
\int_{B}\left|\nabla v_{j}\right|^{2} d x+\int_{B} a_{j} v_{j}^{2} d x=b_{j} \int_{S^{n-1}} v_{j}^{2} d \sigma .
$$

Using (9) and a Poincaré-like inequality, we find there exists $C>0{ }^{1}$

$$
-\int_{B} a_{j} v_{j}^{2} d x \geq C \int_{B} v_{j}^{2} d x .
$$

This leads to a contradiction because $a_{j}(x) \rightarrow 0$ in $C^{0}(\bar{B})$ and $v_{j} \not \equiv 0$.

Case 2. $\frac{1}{C_{M}} \leq\left|\lambda_{i}(c)\right|$. Arguing again by contradiction, let $c_{j} \rightarrow+\infty$ and suppose that $\left|\lambda_{i}\left(c_{j}\right)\right| \rightarrow 0$. As before, the corresponding eigenfunctions $v_{j}$ satisfy (10), where now $b_{j} \rightarrow 1$, because $\mu \sim \kappa c$ and $\left|\lambda_{i}\left(c_{j}\right)\right| \rightarrow 0$. Choosing $v_{j}$ is such a way that $\sup _{B}\left|v_{j}\right|=1$, then (10) yields that $v_{j}$ is bounded in $H^{1}(B)$ and hence $v_{j} \rightarrow v_{0}$ weakly in $H^{1}(B)$. Passing to the limit in

$$
\int_{B} \nabla v_{j} \cdot \nabla w+\int_{B} a_{j} v_{j} w-\int_{S^{n-1}} b_{j} v_{j} w=0, \quad \forall w \in H^{1}(B),
$$

\footnotetext{
${ }^{1}$ in the sequel we will use the same symbol $C$ to denote possibly different positive constants.
} 
it immedately follows that $v_{0}$ satisfies

$$
\begin{cases}\Delta v_{0}=0, & \text { in } B ; \\ \partial_{\nu} v_{0}=v_{0}, & \text { on } S^{n-1} .\end{cases}
$$

The solutions of problem $\left(P_{3}\right)$ are explicitly known, namely they are the linear functions an $B$. We denote by $X$ the vector space of these solutions, which is $n$-dimensional. To complete the proof we will show that $v_{0} \in X$ leads to a contradiction. We know that $\lambda=0$ is an eigenvalue with multiplicity $n$, and the eigenvectors corresponding to $\lambda=0$ are precisely the elements of $T_{z_{\mu}} Z^{c}$. Let $u_{j} \in T_{z_{\mu}\left(c_{j}\right)} Z^{c}$ with $\sup _{B}\left|u_{j}\right|=1$. Then, by using simple computations, one can prove that, up to a subsequence, $u_{j} \rightarrow v$ strongly in $H^{1}(B)$ for some function $v \in X$. We can assume w.l.o.g. that $v=v_{0}$ (the weak limit of $v_{j}$ ), so it follows that $\left(u_{j}, v_{j}\right) \rightarrow\left\|v_{0}\right\|^{2} \neq 0$. But this is not possible, since $v_{j}$ are eigenvectors corresponding to $\lambda_{1}<0$, while $u_{j}$ are eigenvectors corresponding to $\lambda=0$ and hence they are orthogonal.

In conclusion, taking into account of Lemma 2, we can state:

Lemma 3. The unperturbed functional $I_{0}^{c}$ possesses an n-dimensional manifold $Z^{c}$ of critical points, diffeomorphic to a ball of $\mathbb{R}^{n}$. Moreover $I_{0}^{c}$ satisfies the following properties

(i) $I_{0}^{\prime \prime}(z)=I-\mathcal{K}$, where $\mathcal{K}$ is a compact operator for every $z \in Z^{c}$;

(ii) $T_{z} Z^{c}=\operatorname{Ker} D^{2} I_{0}^{c}(z)$ for all $z \in Z^{c}$.

From (i)-(ii) it follows that the restriction of $D^{2} I_{0}^{c}$ to $\left(T_{z} Z^{c}\right)^{\perp}$ is invertible. Moreover, denoting by $L_{c}(z)$ its inverse, for every $M>0$ there exists $C>0$ such that

$$
\left\|L_{c}(z)\right\| \leq C \text { for all } z \in Z^{c} \text { and for all } c>-M \text {. }
$$

\section{The Yamabe like problem}

\subsection{Preliminaries}

Solution s of problem (1) can be found as critical points of the functional $I^{c}$ : $H^{1}(B) \rightarrow \mathbb{R}$ defined in (4).

We recall some formulas from [3] which will be useful for our computations. We denote with $g_{i j}$ the coefficients of the metric $g$ in some local co-ordinates and with $g^{i j}$ the elements of the inverse matrix $\left(g^{-1}\right)_{i j}$. 
The volume element $d V_{g}$ of the metric $g \in \mathcal{G}_{\varepsilon}$, taking into account (2) is

$$
d V_{g}=|g|^{\frac{1}{2}} \cdot d x=(1+O(\varepsilon)) \cdot d x^{2} .
$$

The Christoffel symbols are given by $\Gamma_{i j}^{l}=\frac{1}{2}\left[D_{i} g_{k j}+D_{j} g_{k i}-D_{k} g_{i j}\right] g^{k l}$. The components of the Riemann tensor, the Ricci tensor and the scalar curvature are, respectively

$$
R_{k i j}^{l}=D_{i} \Gamma_{j k}^{l}-D_{j} \Gamma_{i k}^{l}+\Gamma_{i m}^{l} \Gamma_{j k}^{m}-\Gamma_{j m}^{l} \Gamma_{i k}^{m} ; R_{k j}=R_{k l j}^{l} ; \quad R=R_{g}=R_{k j} g^{k j} .
$$

For a smooth function $u$ the components of $\nabla_{g} u$ are $\left(\nabla_{g} u\right)^{i}=g^{i j} D_{j} u$, so

$$
\left(\nabla_{g} u\right)^{i}=\nabla u \cdot(1+O(\varepsilon)) .
$$

From the preceding formulas and from the fact that $g \in \mathcal{G}_{\varepsilon}$ it readily follows that $I^{c}(u)=I_{0}^{c}(u)+O(\varepsilon)$. More precisely, the following lemma holds. The proof is rather technical and is postponed to the Appendix.

Lemma 4. Given $M>0$ there exists $C>0$ such that for $c>-M$ and $g \in \mathcal{G}_{\varepsilon}$ there holds

$$
\begin{gathered}
\left\|\nabla I^{c}(z)\right\| \leq C \cdot \varepsilon \cdot(1+|c|)^{-\frac{n-2}{2}}, \quad \forall z \in Z^{c} ; \\
\left\|D^{2} I^{c}(z)-D^{2} I_{0}^{c}(z)\right\| \leq C \cdot \varepsilon, \quad \forall z \in Z^{c} \\
\left\|I^{c}(z+w)-I^{c}\left(z+w^{\prime}\right)\right\| \leq C \cdot(1+|c|) \cdot \\
\left(\varepsilon+\rho^{\frac{2}{n-2}}\right) \cdot\left\|w-w^{\prime}\right\|, \quad \forall z \in Z^{c}, w, w^{\prime} \in H^{1}(B), \quad \forall\|w\|,\left\|w^{\prime}\right\| \leq \rho \\
\left(1+\|u\|^{\frac{4}{n-2}}+\|w\|^{\frac{4}{n-2}}+|c| \cdot\|u\|^{\frac{2}{n-2}}+|c| \cdot\|w\| \frac{2}{n-2}\right), \quad \forall u, w \in H^{1}(B) .
\end{gathered}
$$

Moreover, if $\|u\|$ is uniformly bounded and if $\|w\| \leq 1$ there results

$$
\left\|D^{2} I^{c}(u+w)-D^{2} I^{c}(u)\right\| \leq C \cdot(1+|c|) \cdot\|w\|^{\frac{2}{n-2}} .
$$




\subsection{A finite dimensional reduction}

The aim of this section is to perform a finite dimensional reduction, using Lemma 3. Arguments of this kind has been emploied, e.g. in [1]. The first step is to construct, for $g \in \mathcal{G}_{\varepsilon}$, a perturbed manifold $Z_{g}^{c} \simeq Z^{c}$ which is a natural constraint for $I^{c}$, namely: if $u \in Z_{g}^{c}$ and $\left.\nabla I^{c}\right|_{Z_{g}^{c}}(u)=0$ then $\nabla I^{c}(u)=0$.

For brevity, we denote by $\left.\dot{z} \in H^{1}(B)\right)^{n}$ an orthonormal $n$-tuple in $T_{z} Z^{c}$. Moreover, if $\alpha \in \mathbb{R}^{n}$ we set $\alpha \dot{z}=\sum \alpha_{i} \dot{z}_{i}$.

Proposition 1. Given $M>0$, there exist $\varepsilon_{0}, C>0$, such that $\forall c>-M$, $\forall z \in Z^{c}$

$\forall \varepsilon \leq \varepsilon_{0}$ and $\forall g \in \mathcal{G}_{\varepsilon}$ there are

$C^{1}$ functions $w=w(z, g, c) \in H^{1}(B)$ and $\alpha=\alpha(z, g, c) \in \mathbb{R}^{n}$ such that the following properties hold

(i) $\quad w$ is orthogonal to $T_{z} Z^{c} \quad \forall z \in Z^{c}$, i.e. $(w, \dot{z})=0$;

(ii) $\nabla I^{c}(z+w)=\alpha \dot{z} \quad \forall z \in Z^{c}$;

(iii) $\|(w, \alpha)\| \leq C \cdot \varepsilon \cdot(1+|c|)^{-\frac{n-2}{2}} \quad \forall z \in Z^{c}$.

Furthermore, from (i)-(ii) it follows that

(iv) the manifold $Z_{g}^{c}=\left\{z+w(z, g, c) \mid z \in Z^{c}\right\}$ is a natural constraint for $I^{c}$.

Proof. Let us define ${ }^{3} H_{g}: Z^{c} \times H^{1}(B) \times \mathbb{R}^{n} \rightarrow H^{1}(B) \times \mathbb{R}^{n}$ by setting

$$
H_{g}(z, w, \alpha)=\left(\begin{array}{c}
\nabla I^{c}(z+w)-\alpha \dot{z} \\
(w, \dot{z})
\end{array}\right) .
$$

With this notation, the unknown $(w, \alpha)$ can be implicitly defined by the equation $H_{g}(z, w, \alpha)=(0,0)$. Setting $R_{g}(z, w, \alpha)=H_{g}(z, w, \alpha)-\partial_{(w, \alpha)} H_{g}(z, 0,0)$ $[(w, \alpha)]$ we have that

$$
H_{g}(z, w, \alpha)=0 \quad \Leftrightarrow \quad \partial_{(w, \alpha)} H_{g}(z, 0,0)[(w, \alpha)]+R_{g}(z, w, \alpha)=0 .
$$

Let $H_{0}=H_{g_{0}}$. From (11) it follows easily that $\partial_{(w, \alpha)} H_{0}(z, 0,0)$ is invertible uniformly w.r.t. $z \in Z^{c}$ and $c>-M$. Moreover using (16) it turns out that for $\varepsilon_{0}$ sufficiently small and for $\varepsilon \leq \varepsilon_{0}$ also the operator $\partial_{(w, \alpha)} H_{g}(z, 0,0)$ is invertible and has uniformly bounded inverse, provided $g \in \mathcal{G}_{\varepsilon}$. Hence, for such $g$ there results

$$
\begin{aligned}
H_{g}(z, w, \alpha) & =0 \Leftrightarrow(w, \alpha)=F_{z, g}(w, \alpha) \\
& :=-\left(\partial_{(w, \alpha)} H_{g}(z, 0,0)\right)^{-1} R_{g}(z, w, \alpha) .
\end{aligned}
$$

We prove the Proposition by showing that the map $F_{z, g}$ is a contraction in some ball $B_{\rho}=\left\{(w, \alpha) \in H^{1}(B) \times \mathbb{R}^{n}:\|w\|+|\alpha| \leq \rho\right\}$, with $\rho$ of order

\footnotetext{
${ }^{3} H$ depends also on $c$, but such a dependence will be understood.
} 
$\rho \sim \varepsilon \cdot(1+|c|)^{-\frac{n-2}{2}}$. We first show that there exists $C>0$ such that for all $(w, \alpha),\left(w^{\prime}, \alpha^{\prime}\right) \in B_{\rho}$, all $z \in Z^{c}$ and all $g \in \mathcal{G}_{\varepsilon}$, there holds

$$
\left\{\begin{array}{l}
\left\|F_{z, g}(w, \alpha)\right\| \leq C \cdot\left(\varepsilon \cdot(1+|c|)^{-\frac{n-2}{2}}+(1+|c|) \cdot \rho^{\frac{n}{n-2}}\right), \\
\left\|F_{z, g}\left(w^{\prime}, \alpha^{\prime}\right)-F_{z, g}(w, \alpha)\right\| \leq C \cdot(1+|c|) \cdot \rho^{\frac{2}{n-2}} \cdot\left\|(w, \alpha)-\left(w^{\prime}, \alpha^{\prime}\right)\right\| .
\end{array}\right.
$$

Condition (20) is equivalent to the following two inequalities

$$
\begin{gathered}
\left\|\nabla I^{c}(z+w)-D^{2} I^{c}(z)[w]\right\| \leq C \cdot\left(\varepsilon \cdot(1+|c|)^{-\frac{n-2}{2}}+(1+|c|) \cdot \rho^{\frac{2}{n-2}}\right) \\
\left\|\left(\nabla I^{c}(z+w)-D^{2} I^{c}(z)[w]\right)-\left(\nabla I^{c}\left(z+w^{\prime}\right)-D^{2} I^{c}(z)\left[w^{\prime}\right]\right)\right\| \leq \\
C \cdot(1+|c|) \cdot \rho^{\frac{2}{n-2}} \cdot\left\|(w, \alpha)-\left(w^{\prime}, \alpha^{\prime}\right)\right\| .
\end{gathered}
$$

Let us first prove (21). There holds

$$
\begin{aligned}
\nabla I^{c}(z+w)-D^{2} I^{c}(z)[w] & =\nabla I^{c}(z+w)-\nabla I^{c}(z)+\nabla I^{c}(z)-D^{2} I^{c}(z)[w] \\
& =\nabla I^{c}(z)+\int_{0}^{1}\left(D^{2} I^{c}(z+s w)-D^{2} I^{c}(z)\right)[w] d s .
\end{aligned}
$$

Hence it turns out that

$$
\left\|\nabla I^{c}(z+w)-D^{2} I^{c}(z)[w]\right\| \leq \nabla I^{c}(z)+\|w\| \cdot \sup _{s \in[0,1]}\left\|D^{2} I^{c}(z+s w)-D^{2} I^{c}(z)\right\| .
$$

Using (19) we have

$$
\left\|\nabla I^{c}(z+w)-D^{2} I^{c}(z)[w]\right\| \leq \nabla I^{c}(z)+C \cdot(1+|c|) \cdot \rho^{\frac{n}{n-2}} .
$$

Hence from (15) we deduce that

$$
\left\|\nabla I^{c}(z+w)-D^{2} I^{c}(z)[w]\right\| \leq C \cdot\left(\varepsilon \cdot(1+|c|)^{-\frac{n-2}{2}}+(1+|c|) \cdot \rho^{\frac{n}{n-2}}\right),
$$

and (21) follows. We turn now to (22). There holds

$$
\begin{aligned}
\| \nabla I^{c}(z+w) & -\nabla I^{c}\left(z+w^{\prime}\right)-D^{2} I^{c}(z)\left[w-w^{\prime}\right] \| \\
& =\left\|\int_{0}^{1}\left(D^{2} I^{c}\left(z+w+s\left(w^{\prime}-w\right)\right)-D^{2} I^{c}(z)\right)\left[w^{\prime}-w\right] d s\right\| \\
& \leq \sup _{s \in[0,1]}\left\|D^{2} I^{c}\left(z+w+s\left(w^{\prime}-w\right)\right)-D^{2} I^{c}(z)\right\| \cdot\left\|w^{\prime}-w\right\| .
\end{aligned}
$$

Using again (19), and taking $\|w\|,\left\|w^{\prime}\right\| \leq \rho$ we have that

$$
\left\|D^{2} I^{c}\left(z+w^{\prime}+s\left(w-w^{\prime}\right)\right)-D^{2} I^{c}(z)\right\| \leq C \cdot(1+|c|) \cdot \rho^{\frac{2}{n-2}},
$$


proving (22). Taking $\rho=2 C \cdot \varepsilon \cdot(1+|c|)^{-\frac{n-2}{2}}$ and $\varepsilon \leq \varepsilon_{0}$, with $\varepsilon_{0}$ sufficiently small, there results

$$
\left\{\begin{array}{l}
C \cdot\left(\varepsilon \cdot(1+|c|)^{-\frac{n-2}{2}}+(1+|c|) \cdot \rho^{\frac{n}{n-2}}\right)<\rho, \\
C \cdot(1+|c|) \cdot \rho^{\frac{2}{n-2}}<1 .
\end{array}\right.
$$

Then $F_{z, g}$ is a contraction in $B_{\rho}$ and hence $H_{g}=0$ has a unique solution $w=$ $w(z, g, c), \alpha=\alpha(z, g, c)$ with $\|(w, \alpha)\| \leq 2 C \cdot \varepsilon \cdot(1+|c|)^{-\frac{n-2}{2}}$.

Remark 1. In general, the preceding arguments give rise to the following result, see [1]. Let $I_{\varepsilon}(u)=I_{0}(u)+O(\varepsilon)$ denote a $C^{2}$ functional and suppose that $I_{0}$ has an $n$-dimensional manifold $Z$ of critical points satisfying $(i)-(i i)$ of Lemma 3. Then for $|\varepsilon|$ small there exists a unique $w=w_{\varepsilon}(z)$ satisfying $(i)-(i i)-(i i i)$ of Proposition 1. Furthermore, the manifold $Z_{\varepsilon}=\left\{z+w_{\varepsilon}(z): z \in Z\right\}$ is a natural constraint for $I_{\varepsilon}$. Hence any critical point of $I_{\varepsilon}\left(z+w_{\varepsilon}(z)\right), z \in Z$ is a critical point of $I_{\varepsilon}$.

\subsection{Proof of Theorem 1}

Throughout this section we will take $\varepsilon$ and $c$ is such a way that Proposition 1 applies. The main tool to prove Theorem 1 is the following Proposition

Proposition 2. There results

$$
\lim _{\mu \rightarrow 0} I^{c}\left(z_{\mu, \xi}+w_{g}\left(z_{\mu, \xi}\right)\right)=b_{c}, \quad \text { uniformly for } \xi \text { satisfying (5). }
$$

Hence $\left.I^{c}\right|_{Z_{g}^{c}}$ can be continuously extended to $\partial Z_{g}^{c}$ by setting

$$
\left.I^{c}\right|_{\partial Z_{g}^{c}}=b_{c} \text {. }
$$

Postponing the proof of Proposition 2, it is immediate to deduce Theorem 1.

Proof of Theorem 1. The extended functional $I^{c}$ has a critical point on the compact manifold $Z_{g}^{c} \cup \partial Z_{g}^{c}$. From (24) it follows that either $I^{c}$ is identically constant or it achieves the maximum or the minimum in $Z_{g}^{c}$. In any case $I^{c}$ has a critical point on $Z_{g}^{c}$. According to Proposition 1, such a critical point gives rise to a solution of $(Y)$.

In order to prove Proposition 2 we prefer to reformulate $(Y)$ in a more convenient form using the stereographic projection $\sigma_{p}$, trough an appropriate point $p \in \partial S_{+}^{n}$, see Remark 3. In this way the problem reduces to study an elliptic equation in $\mathbb{R}_{+}^{n}$, where calculation are easier. More precisely, let $\tilde{g}_{i j}: \mathbb{R}_{+}^{n} \rightarrow \mathbb{R}$ be the components of the metric $g$ in $\sigma_{p}$-stereographic co-ordinates, and let

$$
\bar{g}_{i j}=\left(\frac{1+|x|^{2}}{2}\right)^{2} \tilde{g}_{i j} .
$$


Then problem $(Y)$ is equivalent to find solutions of

$$
\left\{\begin{array}{l}
-4 \frac{(n-1)}{(n-2)} \Delta_{\bar{g}} u+R_{\bar{g}} u=u^{\frac{n+2}{n-2},} \quad \text { in } \mathbb{R}_{+}^{n} ; \\
\frac{2}{(n-2)} \partial_{\nu_{\bar{g}}} u+h_{\bar{g}} u=c u^{\frac{n}{n-2}}, \quad \text { on } \partial \mathbb{R}_{+}^{n}=\mathbb{R}^{n-1}, \\
u>0, \quad u \in \mathcal{D}^{1,2}\left(\mathbb{R}_{+}^{n}\right),
\end{array}\right.
$$

where the symbols have obvious meaning. Solutions of problem $(\bar{Y})$ can be found as critical points of the functional $f_{\bar{g}}: \mathcal{D}^{1,2}\left(\mathbb{R}_{+}^{n}\right) \rightarrow \mathbb{R}$ defined in the following way

$$
\begin{aligned}
f_{\bar{g}}(u) & =2 \frac{(n-1)}{(n-2)} \int_{\mathbb{R}_{+}^{n}}\left|\nabla_{\bar{g}} u\right|^{2} d V_{\bar{g}}+\frac{1}{2} \int_{\mathbb{R}_{+}^{n}} R_{\bar{g}} u^{2} d V_{\bar{g}}-\frac{1}{2^{*}} \int_{\mathbb{R}_{+}^{n}} u^{2^{*}} d V_{\bar{g}} \\
& +(n-1) \int_{\partial \mathbb{R}_{+}^{n}} h_{\bar{g}} u^{2} d \sigma_{\bar{g}}-c(n-2) \int_{\partial \mathbb{R}_{+}^{n}}|u|^{2 \frac{n-1}{n-2}} d \sigma_{\bar{g}} .
\end{aligned}
$$

In general the transformation $(\bar{g})$ induces an isometry between $H^{1}(B)$ and $\mathcal{D}^{1,2}\left(\mathbb{R}_{+}^{n}\right)$ given by

$$
\begin{aligned}
u(x) \mapsto \bar{u}(x) & :=\left(\frac{2}{\left(x^{\prime}\right)^{2}+\left(x_{n}+1\right)^{2}}\right)^{\frac{n-2}{2}} \\
& \times u\left(\frac{2 x^{\prime}}{\left(x^{\prime}\right)^{2}+\left(x_{n}+1\right)^{2}}, \frac{\left(x^{\prime}\right)^{2}+x_{n}^{2}-1}{\left(x^{\prime}\right)^{2}+\left(x_{n}+1\right)^{2}}\right),
\end{aligned}
$$

where $x^{\prime}=\left(x_{1}, \ldots, x_{n-1}\right)$.

It turns out that

$$
f_{\bar{g}}(\bar{u})=I^{c}(u)
$$

as well as

$$
\nabla f_{\bar{g}}(\bar{u})=\nabla I^{c}(u) .
$$

In particular this implies that $u$ solves $(Y)$ if and only if $\bar{u}$ is a solution of $(\bar{Y})$.

Furthermore, there results

- $g_{0}$ corresponds to the trivial metric $\delta_{i j}$ on $\mathbb{R}_{+}^{n}$;

- $z_{0}$ corresponds to $\bar{z}_{0} \in \mathcal{D}^{1,2}\left(\mathbb{R}_{+}^{n}\right)$ given by

$$
\bar{z}_{0}(x)=z_{0}\left(x-\left(0, a_{0} c\right)\right), \quad x \in \mathbb{R}_{+}^{n} ; \quad a_{0}=\frac{\kappa}{2} ;
$$

- $Z^{c}$ corresponds to $\bar{Z}^{c}$ given by

$$
\bar{Z}^{c}=\left\{\bar{z}_{\mu, \xi^{\prime}}:=\mu^{-\frac{n-2}{2}} z_{0}\left(\frac{x-\left(\xi^{\prime}, a_{0} c \mu\right)}{\mu}\right), \mu>0, \xi^{\prime} \in \mathbb{R}^{n-1}\right\} .
$$


Let us point out that the manifold $\bar{Z}^{c}$ is nothing but $\tau_{p} \circ \tau_{S}^{-1} Z^{c}$ (see Notations).

From the preceding items it follows that the equation

$$
\nabla f_{\bar{g}}(\bar{z}+\bar{w}) \in T_{\bar{z}} \bar{Z}^{c}
$$

have a unique solution $\bar{w} \perp T_{\bar{z}} \bar{Z}^{c}$ and there results

$$
\bar{w}_{\bar{g}}(\bar{z})=\overline{w_{g}(z)} .
$$

From this and (25) it follows

$$
I^{c}\left(z+w_{g}(z)\right)=f_{\bar{g}}\left(\bar{z}+\bar{w}_{\bar{g}}(\bar{z})\right) .
$$

Let us now introduce the metric $\bar{g}^{\delta}(x):=\bar{g}(\delta x), \delta>0$ and let $f_{\bar{g}^{\delta}}: \mathcal{D}^{1,2}\left(\mathbb{R}_{+}^{n}\right) \rightarrow$ $\mathbb{R}$ be the corresponding Euler functional. For all $u \in \mathcal{D}^{1,2}\left(\mathbb{R}_{+}^{n}\right)$ there results

$$
f_{\bar{g} \delta}(u)=f_{\bar{g}}\left(\delta^{\frac{2-n}{2}} u\left(\delta^{-1} x\right)\right) .
$$

Introducing the linear isometry $T_{\delta}: \mathcal{D}^{1,2}\left(\mathbb{R}_{+}^{n}\right) \rightarrow \mathcal{D}^{1,2}\left(\mathbb{R}_{+}^{n}\right)$ defined by $T_{\delta}(u):=$ $\delta^{-\frac{n-2}{2}} u(x / \delta)$ this becomes

$$
f_{\bar{g} \delta}(u)=f_{\bar{g}}\left(T_{\delta} u\right),
$$

Furthermore, for all $u \in \mathcal{D}^{1,2}\left(\mathbb{R}_{+}^{n}\right)$ one has

$$
\begin{aligned}
\nabla f_{\bar{g}}(u) & =T_{\delta} \nabla f_{\bar{g}^{\delta}}\left(T_{\delta}^{-1} u\right) \\
D^{2} f_{\bar{g}}(u)[v, w] & =D^{2} f_{\bar{g}^{\delta}}\left(T_{\delta}^{-1} u\right)\left[T_{\delta}^{-1} v, T_{\delta}^{-1} w\right] .
\end{aligned}
$$

Arguing as above, there exists $\bar{w}_{\bar{g}^{\delta}}\left(\bar{z}_{0}\right) \in\left(T_{\bar{z}_{0}} \bar{Z}^{c}\right)^{\perp}$ such that

$$
\nabla f_{\bar{g}^{\delta}}\left(\bar{z}_{0}+\bar{w}_{\bar{g}^{\delta}}\right) \in T_{\bar{z}_{0}} \bar{Z}^{c} .
$$

and there results

$$
\bar{w}_{\bar{g}^{\delta}}\left(\bar{z}_{0}\right)(x)=\delta^{\frac{n-2}{2}} \bar{w}_{\bar{g}}\left(\bar{z}_{\delta}\right)(\delta x)
$$

namely

$$
\bar{w}_{\bar{g}}\left(\bar{z}_{\delta}\right)=T_{\delta} \bar{w}_{\bar{g}^{\delta}}\left(\bar{z}_{0}\right) .
$$

Remark 2. From (27), (28), (29) and using the relations between $f_{\bar{g}}$ and $I^{c}$ discussed above, it is easy to check that the estimates listed in Lemma 4 hold true, substituting $I^{c}$ with $f_{\bar{g} \delta}$ and $z$ with $\bar{z}$. A similar remark holds for Proposition 1 . 
We are interested to the behaviour of $f_{\bar{g}^{\delta}}$ as $\delta \rightarrow 0$. To this purpose, we set

$$
\begin{aligned}
f_{\bar{g}(0)}(u)= & \int_{\mathbb{R}_{+}^{n}}\left(2 \frac{(n-1)}{(n-2)} \sum_{i, j} \bar{g}^{i j}(0) D_{i} u D_{j} u-\frac{1}{2^{*}}|u|^{2^{*}}\right) d V_{\bar{g}(0)} \\
& -c(n-2) \int_{\partial \mathbb{R}_{+}^{n}}|u|^{2 \frac{n-1}{n-2}} d \sigma_{\bar{g}(0)},
\end{aligned}
$$

which is the Euler functional corresponding to the constant metric $\bar{g}(0)$.

Remark 3. Unlike the $\bar{g}^{\delta}$, the metric $\bar{g}(0)$ does not come from a smooth metric on $B$. This is the main reason why it is easier to deal with $(\bar{Y})$ instead of $(Y)$.

Lemma 5. For all $u \in \mathcal{D}^{1,2}\left(\mathbb{R}_{+}^{n}\right)$ there results

$$
\begin{gathered}
\lim _{\delta \rightarrow 0}\left\|\nabla f_{\bar{g}^{\delta}}(u)-\nabla f_{\bar{g}(0)}(u)\right\|=0 ; \\
\lim _{\delta \rightarrow 0} f_{\bar{g}^{\delta}}(u)=f_{\bar{g}(0)}(u) .
\end{gathered}
$$

Proof. For any $v \in \mathcal{D}^{1,2}\left(\mathbb{R}_{+}^{n}\right)$ there holds

$$
\left(\nabla f_{\bar{g}^{\delta}}(u)-\nabla f_{\bar{g}(0)}(u), v\right)=\theta_{1}+\theta_{2}+\theta_{3}+\theta_{4}+\theta_{5},
$$

where

$$
\begin{aligned}
& \theta_{1}=4 \frac{n-1}{n-2}\left(\int_{\mathbb{R}_{+}^{n}} \nabla_{\bar{g}^{\delta}} u \cdot \nabla_{\bar{g}^{\delta}} v d V_{\bar{g}^{\delta}}-\int_{\mathbb{R}_{+}^{n}} \nabla_{\bar{g}(0)} u \cdot \nabla_{\bar{g}(0)} v d V_{\bar{g}(0)}\right) ; \\
& \theta_{2}=\int_{\mathbb{R}_{+}^{n}} R_{\bar{g} \delta} u v d V_{\bar{g}^{\delta}} ; \\
& \theta_{3}=\int_{\mathbb{R}_{+}^{n}}|u|^{\frac{4}{n-2}} u v\left(d V_{\bar{g}^{\delta}}-d V_{\bar{g}(0)}\right) ; \theta_{4}=2(n-1) \int_{\partial \mathbb{R}^{n-1}} h_{\bar{g} \delta} u v d \sigma_{\bar{g}^{\delta}} ; \\
& \theta_{5}=2 c(n-1)\left(\int_{\partial \mathbb{R}_{+}^{n}}|u|^{\frac{2}{n-2}} u v d \sigma_{\bar{g}^{\delta}}-\int_{\partial \mathbb{R}_{+}^{n}}|u|^{\frac{2}{n-2}} u v d \sigma_{\bar{g}(0)}\right) .
\end{aligned}
$$

Using the Dominated Convergence Theorem and the integrability of $|\nabla u|^{2}$ and of $|u|^{2^{*}}$, it is easy to show that $\theta_{1}, \theta_{3}$ and $\theta_{5}$ converge to zero. As far as $\theta_{2}$ is concerned, we first note that the bilinear form $(u, v) \rightarrow \int_{\mathbb{R}_{+}^{n}} R_{\bar{g}} u v d V_{\bar{g}}$ is uniformly bounded for $\bar{g} \in \overline{\mathcal{G}}_{\varepsilon}$, so it turns out that given $\eta>0$ there exists $u_{\eta} \in C_{c}^{\infty}\left(\overline{\mathbb{R}}_{+}^{n}\right)$ such that

$$
\left|\int_{\mathbb{R}_{+}^{n}} R_{\bar{g}^{\delta}} u v d V_{\bar{g}^{\delta}}-\int_{\mathbb{R}_{+}^{n}} R_{\bar{g}^{\delta}} u_{\eta} v d V_{\bar{g}^{\delta}}\right| \leq \eta \cdot\|v\| ; \quad \forall v \in \mathcal{D}^{1,2}\left(\mathbb{R}_{+}^{n}\right) .
$$


Hence, since it is $R_{\bar{g} \delta}\left(\delta^{-1} x\right)=\delta^{2} R_{\bar{g}}(x)$ (see (13)), it follows that for $\delta$ sufficiently small

$$
\left|\int_{\mathbb{R}_{+}^{n}} R_{\bar{g}^{\delta}} u_{\eta} v d V_{\bar{g}^{\delta}}\right| \leq \delta^{2}\left\|R_{\bar{g}}\right\|_{L^{\infty}(B)}\left\|u_{\eta}\right\|_{\infty} \int_{\operatorname{supp}\left(u_{\eta}\right)}|v|=o(1) \cdot\|v\| .
$$

So, using (33) and the arbitrarity of $\eta$, one deduces that $\theta_{2}=o(1) \cdot\|v\|$. Similar computations hold for the term $\theta_{4}$. In the same way one can prove also (32).

We need a more complete description of $\bar{w}^{0}(\bar{z})$. For this, according to Remark 3 , we shall study the functional $f_{\bar{g}(0)}$ in a direct fashion. If $g \in \mathcal{G}_{\varepsilon}$ then the constant metric $\bar{g}(0)$ on $\mathbb{R}_{+}^{n}$ satisfies $\|\bar{g}(0)-I d\|_{\infty}=O(\varepsilon)$ and thus $f_{\bar{g}(0)}$ can be seen as a perturbation of the functional

$$
f_{0}(u)=2 \frac{(n-1)}{(n-2)} \int_{\mathbb{R}_{+}^{n}}|\nabla u|^{2} d V_{0}-\frac{1}{2^{*}} \int_{\mathbb{R}_{+}^{n}} u^{2^{*}} d V_{0}-c(n-2) \int_{\partial \mathbb{R}_{+}^{n}}|u|^{2 \frac{n-1}{n-2}} d \sigma_{0},
$$

corresponding to the trivial metric $\delta_{i j}$.

Then the procedure used in Sect. 3.2 yields to find $\bar{w}^{0}(\bar{z})$ such that

(j) $\bar{w}^{0}(\bar{z})$ is orthogonal to $T_{\bar{z}} \bar{Z}^{c}$;

(jj) $\nabla f_{\bar{g}(0)}\left(\bar{z}+\bar{w}^{0}(\bar{z})\right) \in T_{\bar{z}} \bar{Z}^{c}$;

(jjj) $\left\|\bar{w}^{0}(\bar{z})\right\| \leq C \cdot \varepsilon \cdot(1+|c|)^{-\frac{n-2}{2}} \forall \bar{z} \in \bar{Z}^{c}$.

The following Lemma proves that a property stronger than $(j j)$ holds.

Lemma 6. For all $\bar{z} \in \bar{Z}^{c}$ there results

$$
\nabla f_{\bar{g}(0)}\left(\bar{z}+\bar{w}_{\bar{g}(0)}(\bar{z})\right)=0 .
$$

Hence $\bar{z}+\bar{w}_{\bar{g}(0)}(\bar{z})$ solves

$$
\begin{cases}-4 \frac{(n-1)}{(n-2)} \sum_{i, j=1}^{n} \bar{g}^{i j}(0) D_{i j}^{2} u=u^{\frac{n+2}{n-2}} & \text { in } \mathbb{R}_{+}^{n} \\ \frac{2}{(n-2)} \frac{\partial u}{\partial \bar{v}}=c u^{\frac{n}{n-2}} & \text { on } \partial \mathbb{R}_{+}^{n} .\end{cases}
$$

Here $\bar{v}$ is the unit normal vector to $\partial \mathbb{R}_{+}^{n}$ with respect to $\bar{g}(0)$, namely

$$
\bar{g}(0)(\bar{v}, \bar{v})=1 ; \quad \bar{g}(0)(\bar{v}, v)=0, \quad \forall v \in \partial \mathbb{R}_{+}^{n} .
$$

Proof. The Lemma is a simple consequence of the invariance of the functional under the transformation $T_{\mu, \xi^{\prime}}: \mathcal{D}^{1,2}\left(\mathbb{R}_{+}^{n}\right) \rightarrow \mathcal{D}^{1,2}\left(\mathbb{R}_{+}^{n}\right)$ defined in the following way

$$
T_{\mu, \xi^{\prime}}(u)=\mu^{-\frac{n-2}{2}} u\left(\frac{x-\left(\xi^{\prime}, 0\right)}{\mu}\right) .
$$

This can be achieved with an elementary computation. It then follows that

$$
\bar{w}_{\bar{g}(0)}\left(\bar{z}_{\mu, \xi^{\prime}}\right)=T_{\mu, \xi^{\prime}}\left(\bar{w}_{\bar{g}(0)}\left(\bar{z}_{0}\right)\right), \quad \text { for all } \mu, \xi^{\prime} .
$$


Hence, from the invariance of $f_{\bar{g}(0)}$, it turns out that

$f_{\bar{g}(0)}\left(\bar{z}_{\mu, \xi^{\prime}}+\bar{w}_{\bar{g}(0)}\left(\bar{z}_{\mu, \xi^{\prime}}\right)\right)=f_{\bar{g}(0)}\left(T_{\mu, \xi^{\prime}}\left(\bar{z}_{0}+\bar{w}_{\bar{g}(0)}\left(\bar{z}_{0}\right)\right)\right)=f_{\bar{g}(0)}\left(\bar{z}_{0}+\bar{w}_{\bar{g}(0)}\left(\bar{z}_{0}\right)\right)$.

Since $f_{\bar{g}(0)}\left(\bar{z}_{\mu, \xi^{\prime}}+\bar{w}_{\bar{g}(0)}\left(\bar{z}_{\mu, \xi^{\prime}}\right)\right)$ is a constant function then, according to $(j)-$ $(j j)$, any $\bar{z}+\bar{w}_{\bar{g}(0)}(\bar{z})$ is a critical point of $f_{\bar{g}(0)}$, proving the lemma.

Let us introduce some further notation: $\bar{G}$ denotes the matrix $\bar{g}_{i j}(0), v_{\bar{g}(0)}$ is the outward unit normal to $\partial \mathbb{R}_{+}^{n}$ with respect to $\bar{g}_{i j}(0)$, and $e_{1}, \ldots, e_{n}$ is the standard basis of $\mathbb{R}^{n}$.

Lemma 7. The solutions u of problem (35) are, up to dilations and translations, of the form

$$
u=\bar{z}_{0}(A x),
$$

where A is a matrix which satisfies

$$
A \bar{G}^{-1} A^{T}=I, \quad \nu_{\bar{g}(0)}=\sum_{j}\left(A^{-1}\right)_{j n} e_{j}
$$

In particular, up to dilations, one has that

$$
\bar{z}_{0}+\bar{w}_{\bar{g}(0)}\left(\bar{z}_{0}\right)=\bar{z}_{0}(A \cdot) .
$$

Proof. First of all we prove the existence of a matrix $A$ satisfying (36). The first equality simply means that the bilinear form represented by the matrix $\bar{G}^{-1}$ can be diagonalized, and this is standard. The matrix $A$ which satisfies the first equation in (36) is defined uniquely up to multiplication on the left by an orthogonal matrix. Let $\left(x_{1}, \ldots, x_{n}\right)$ be the co-ordinates with respect to the standard basis $\left(e_{1}, \ldots, e_{n}\right)$ of $\mathbb{R}^{n}$, let $\left(f_{1}, \ldots, f_{n}\right)$ be the basis given by $\mathbf{f}=\left(\mathbf{A}^{-\mathbf{1}}\right)^{\mathbf{T}} \mathbf{e}$, and let $\left(y_{1}, \ldots, y_{n}\right)$ be the co-ordinates with respect to this new basis. This implies the relation between the co-ordinates $x=A y$ and the first of (36) implies that the bilinear form $\bar{g}^{i j}(0)$ is diagonal with respect to $y_{1}, \ldots y_{n}$. Moreover, by the transitive action of $O(n)$ over $S^{n-1}$ we can ask that $f_{n}=v$; this is exactly the second equation in (36). In this way the matrix $A$ is determined up to multiplication on the left by $O(n-1)$.

We now prove that the function $\tilde{z}_{0}=\bar{z}_{0}(A x)=\bar{z}_{0}(y)$ is a solution of (35). First of all, since $v_{\bar{g}(0)}$ is $\bar{g}(0)$-orthogonal to $\partial \mathbb{R}^{n-1}$, the domain $x_{n}>0$ coincides with $y_{n}>0$ and the equation in the interior is, by formula (36)

$$
-4 \frac{(n-1)}{(n-2)} \sum_{i, j=1}^{n} D_{x_{i} x_{j}}^{2} \tilde{z}_{0}(x)=-4 \frac{(n-1)}{(n-2)} \sum_{i, j} \bar{g}^{i j} A_{l i} A_{k j} D_{y_{k} y_{l}}^{2} \bar{z}_{0}(A y)=\tilde{z}_{0}^{\frac{n+2}{n-2}}(x) .
$$


Moreover, since $v=f_{n}=\sum_{j}\left(A^{-1}\right)_{n j}^{T} e_{j}=\sum_{j}\left(A^{-1}\right)_{j n} e_{j}$, it turns out that on $\partial \mathbb{R}_{+}^{n}$

$$
\begin{aligned}
\frac{\partial \tilde{z}_{0}}{\partial \bar{v}}(x) & =\sum_{j}\left(A^{-1}\right)_{j n} D_{x_{j}} \bar{z}_{0}(A y) \\
& =\sum_{j, k}\left(A^{-1}\right)_{j n} A_{k j} D_{y_{k}} \bar{z}_{0}(A y)=D_{y_{n}} \bar{z}_{0}(A y)=c \tilde{z}_{0}^{\frac{n}{n-2}}(x) .
\end{aligned}
$$

Hence also the boundary condition is satisfied. Moreover, the function $\bar{z}_{0} \in$ $\mathcal{D}^{1,2}\left(\mathbb{R}_{+}^{n}\right)$ is the unique solution up to dilation and translation of problem $(\bar{Y})$ with $\bar{g}_{i j}=I d$, see [14]. As pointed out before, if $A$ and $A^{\prime}$ are two matrices satisfying (36), they differ up to $O(n-1)$. Then it is easy to check that $\bar{z}_{0}(A x)=\bar{z}_{0}\left(A^{\prime} x\right)$ and hence $\tilde{z}_{0}$ is unique up to dilation and translation. This concludes the proof.

Corollary 1. The quantity $f_{\bar{g}(0)}\left(\bar{z}_{0}+\bar{w}^{0}\left(\bar{z}_{0}\right)\right)$ is independent of $\bar{g}(0)$. Precisely one has:

$$
f_{\bar{g}(0)}\left(\bar{z}_{0}+\bar{w}^{0}\left(\bar{z}_{0}\right)\right)=b_{c} .
$$

Proof. There holds

$$
\begin{aligned}
& f_{\bar{g}(0)}\left(\bar{z}_{0}+\bar{w}_{\bar{g}(0)}\left(\bar{z}_{0}\right)\right) \\
& =2 \frac{(n-1)}{(n-2)} \int_{\mathbb{R}_{+}^{n}} \sum_{i, j, k, l} \bar{g}^{i j}(0) A_{k i} A_{l j} D_{k} \bar{z}_{0}(A y) D_{l} \bar{z}_{0}(A y) d V_{\bar{g}(0)}(y) \\
& -\frac{1}{2^{*}} \int_{\mathbb{R}_{+}^{n}}\left|\bar{z}_{0}(A y)\right|^{2^{*}} d V_{\bar{g}(0)}(y)-c(n-2) \int_{\partial \mathbb{R}_{+}^{n}}\left|\bar{z}_{0}(A y)\right|^{2 \frac{n-1}{n-2}} d \sigma_{\bar{g}(0)}(y) .
\end{aligned}
$$

Using the change of variables $x=A y$, and taking into account equations (12) and (36) we obtain the claim. This concludes the proof.

Lemma 8. There holds

$$
\bar{w}_{\bar{g}^{\delta}}\left(\bar{z}_{0}\right) \rightarrow \bar{w}_{\bar{g}(0)} \quad \text { as } \delta \rightarrow 0 .
$$

Proof. Define $\bar{H}^{\delta}: \mathcal{D}^{1,2}\left(\mathbb{R}_{+}^{n}\right) \times \mathbb{R}^{n} \times \bar{Z}^{c} \rightarrow \mathcal{D}^{1,2}\left(\mathbb{R}_{+}^{n}\right) \times \mathbb{R}^{n}$ by setting

$$
\bar{H}^{\delta}(w, \alpha, \bar{z})=\left(\begin{array}{c}
\nabla f_{\bar{g}^{\delta}}\left(\bar{z}+\bar{w}_{\bar{g}(0)}+w\right)-\alpha \dot{\bar{z}} \\
(w, \dot{\bar{z}})
\end{array}\right) .
$$

One has that

$$
\nabla f_{\bar{g}^{\delta}}\left(\bar{z}+\bar{w}_{\bar{g}(0)}+w\right)=\nabla f_{\bar{g}^{\delta}}\left(\bar{z}+\bar{w}_{\bar{g}(0)}\right)+D^{2} f_{\bar{g}^{\delta}}\left(\bar{z}+\bar{w}_{\bar{g}(0)}\right)[w]+\vartheta(w)
$$

where

$$
\vartheta(w):=\int_{0}^{1}\left(D^{2} f_{\bar{g}^{\delta}}\left(\bar{z}+\bar{w}_{\bar{g}(0)}+s w\right)-D^{2} f_{\bar{g}^{\delta}}\left(\bar{z}+\bar{w}_{\bar{g}(0)}\right)\right)[w] d s .
$$


Recall that $D^{2} f_{\bar{g}^{\delta}}(\bar{z})$ is invertible on $\left(T_{\bar{z}} \bar{Z}^{c}\right)^{\perp}$. Since $\bar{w}_{\bar{g}(0)}$ satisfies $(j j j)$, then also $D^{2} f_{\bar{g}^{\delta}}\left(\bar{z}+\bar{w}_{\bar{g}(0)}\right)$ is invertible on $\left(T_{\bar{Z}} \bar{Z}^{c}\right)^{\perp}$. As a consequence, the equation $\nabla f_{\bar{g}^{\delta}}\left(\bar{z}+\bar{w}_{\bar{g}(0)}+w\right)=0, w \in\left(T_{\bar{z}} \bar{Z}^{c}\right)^{\perp}$ is equivalent, on $\left(T_{\bar{z}} \bar{Z}^{c}\right)^{\perp}$, to

$$
w=-\left(D^{2} f_{\bar{g} \delta}\left(\bar{z}+\bar{w}_{\bar{g}(0)}\right)\right)^{-1}\left[\nabla f_{\bar{g}^{\delta}}\left(\bar{z}+\bar{w}_{\bar{g}(0)}\right)+\vartheta(w)\right]
$$

In addition, by Remark 2, we can use the estimates corresponding to (19) of Lemma 4 and to $(\mathrm{iii})$ of Proposition 1, to infer that

$$
\vartheta(w)=\int_{0}^{1}\left(D^{2} f_{\bar{g}^{\delta}}\left(\bar{z}+\bar{w}_{\bar{g}(0)}+s w\right)-D^{2} f_{\bar{g}^{\delta}}\left(\bar{z}+\bar{w}_{\bar{g}(0)}\right)\right)[w] d s=o(\|w\|) .
$$

Then, repeating the arguments used in Sect. 3.2 with small changes, one can show that the equation $\bar{H}^{\delta}=0$ has a unique solution $w=\omega$ such that

$$
\|\omega\| \leq C \cdot\left\|\nabla f_{\bar{g}^{\delta}}\left(\bar{z}+\bar{w}_{\bar{g}(0)}\right)\right\| .
$$

From (34) and (31) it follows that $\|\omega\| \rightarrow 0$ as $\delta \rightarrow 0$. Since both $\bar{w}_{\bar{g}(0)}+\omega$ and $\bar{w}_{\bar{g}^{\delta}}$ solve (on $\left(T_{\bar{z}} \bar{Z}^{c}\right)^{\perp}$ ) the same equation, we infer by uniqueness that $\bar{w}_{\bar{g}^{\delta}}=\bar{w}_{\bar{g}(0)}+\omega$. Finally, since $\|\omega\| \rightarrow 0$ as $\delta \rightarrow 0$, then (37) follows.

Remark 4. All the preceding discussion has been carried out by taking the stereographic projection $\sigma_{p}$ through an arbitrary $p \in S^{n-1}$. We are interested to the limit (23). When $\mu \rightarrow 0$ then $\xi \rightarrow \bar{\xi}$ for some $\bar{\xi} \in S^{n-1}$ and it will be convenient to choose $p=-\bar{\xi}$.

We are now in position to give:

Proof of Proposition 2. As pointed out in Remark 4, we take $p=-\bar{\xi}$ and use all the preceding results proved so far in this section. With this choice, when $(\mu, \xi) \rightarrow(0, \bar{\xi})$ with $\xi=|\xi| \cdot \bar{\xi}, z_{\mu, \xi}$ corresponds to $\bar{z}_{\mu^{\prime}}:=\bar{z}_{\mu^{\prime}, 0}$, for some $\mu^{\prime} \rightarrow 0$.

Next, in view of (26), we will show that

$$
\lim _{\mu^{\prime} \rightarrow 0} f_{\bar{g}}\left(\bar{z}_{\mu^{\prime}}+\bar{w}_{\bar{g}}\left(\bar{z}_{\mu^{\prime}}\right)\right)=b_{c} .
$$

By Corollary $1, b_{c}=f_{\bar{g}(0)}\left(\bar{z}_{0}+\bar{w}_{\bar{g}(0)}\right)$ and hence we need to prove that

$$
\lim _{\mu^{\prime} \rightarrow 0}\left[f_{\bar{g}}\left(\bar{z}_{\mu^{\prime}}+\bar{w}_{\bar{g}}\left(\bar{z}_{\mu^{\prime}}\right)\right)-f_{\bar{g}(0)}\left(\bar{z}_{0}+\bar{w}_{\bar{g}(0)}\right)\right]=0 .
$$

Using (30), we have

$$
f_{\bar{g}}\left(\bar{z}_{\mu^{\prime}}+\bar{w}_{\bar{g}}\left(\bar{z}_{\mu^{\prime}}\right)\right)=f_{\bar{g}}\left(\bar{z}_{\mu^{\prime}}+T_{\mu^{\prime}} \bar{w}_{\bar{g}^{\mu^{\prime}}}\left(\bar{z}_{0}\right)\right) .
$$


Then we can write

$$
\begin{aligned}
& f_{\bar{g}}\left(\bar{z}_{\mu^{\prime}}+\bar{w}_{\bar{g}}\left(\bar{z}_{\mu u^{\prime}}\right)\right)-f_{\bar{g}(0)}\left(\bar{z}_{0}+\bar{w}_{\bar{g}(0)}\right)=f_{\bar{g}}\left(\bar{z}_{\mu^{\prime}}+T_{\mu^{\prime}} \bar{w}_{\bar{g}^{\mu^{\prime}}}\left(\bar{z}_{0}\right)\right) \\
& =f_{\bar{g}}\left(\bar{z}_{\mu^{\prime}}+T_{\mu^{\prime}} \bar{w}_{\bar{g}^{\prime}}\left(\bar{z}_{0}\right)\right)-f_{\bar{g}}\left(\bar{z}_{\mu^{\prime}}+T_{\mu^{\prime}} \bar{w}_{\bar{g}(0)}\left(\bar{z}_{0}\right)\right) \\
& \quad+\ddot{\mathrm{i}} f_{\bar{g}}\left(\bar{z}_{\mu^{\prime}}+T_{\mu^{\prime}} \bar{w}_{\bar{g}(0)}\left(\bar{z}_{0}\right)\right)-f_{\bar{g}(0)}\left(\bar{z}_{0}+\bar{w}_{\bar{g}(0)}\right) .
\end{aligned}
$$

From (17) with $I^{c}$ substituted by $f_{\bar{g}}$, we infer

$$
\begin{aligned}
& \mid f_{\bar{g}}\left(\bar{z}_{\mu^{\prime}}+T_{\mu^{\prime}} \bar{w}_{\bar{g}^{\mu^{\prime}}}\left(\bar{z}_{0}\right)\right)-f_{\bar{g}}\left(\bar{z}_{\mu^{\prime}}+T_{\mu^{\prime}} \bar{w}_{\bar{g}(0)}\left(\text { ov } z_{0}\right)\right) \mid \\
& \leq C \cdot\left\|T_{\mu^{\prime}} \bar{w}_{\bar{g}^{\mu^{\prime}}}\left(\bar{z}_{0}\right)-T_{\mu^{\prime}} \bar{w}_{\bar{g}(0)}\left(\bar{z}_{0}\right)\right\| \\
& \leq C \cdot\left\|\bar{w}_{\bar{g}^{\mu^{\prime}}}\left(\bar{z}_{0}\right)-\bar{w}_{\bar{g}(0)}\left(\bar{z}_{0}\right)\right\| \\
& =o(1) \text { as } \mu^{\prime} \rightarrow 0 .
\end{aligned}
$$

Using $\bar{z}_{\mu^{\prime}}=T_{\mu^{\prime}} \bar{z}_{0}$ and (27), we deduce

$$
f_{\bar{g}}\left(\bar{z}_{\mu^{\prime}}+T_{\mu^{\prime}} \bar{w}_{\bar{g}^{\mu^{\prime}}}\left(\bar{z}_{0}\right)\right)=f_{\bar{g}}\left(T_{\mu^{\prime}}\left(\bar{z}_{0}+\bar{w}_{\bar{g}^{\mu^{\prime}}}\left(\bar{z}_{0}\right)\right)\right)=f_{\bar{g}^{\mu^{\prime}}}\left(\bar{z}_{0}+\bar{w}_{\bar{g}(0)}\right) .
$$

Finally

$$
\begin{aligned}
& \left|f_{\bar{g}}\left(\bar{z}_{\mu^{\prime}}+T_{\mu^{\prime}} \bar{w}_{\bar{g}^{\mu^{\prime}}}\left(\bar{z}_{0}\right)\right)-f_{\bar{g}(0)}\left(\bar{z}_{0}+\bar{w}_{\bar{g}(0)}\right)\right| \\
& =\left|f_{\bar{g}^{\mu^{\prime}}}\left(\bar{z}_{0}+\bar{w}_{\bar{g}(0)}\right)-f_{\bar{g}(0)}\left(\bar{z}_{0}+\bar{w}_{\bar{g}(0)}\right)\right| \rightarrow 0,
\end{aligned}
$$

according to Lemma 5. Since the above arguments can be carried out uniformly with respect to $\xi^{\prime} \in S^{n-1}$, the proof is completed.

\section{The scalar curvature problem}

In this section the value of $c$ is fixed. Therefore its dependence will be omitted. So we will write $I_{\varepsilon}$ instead of $I_{\varepsilon}^{c}, I_{0}$ instead of $I_{0}^{c}$, etc.

\subsection{The abstract setting}

Solutions of problem $\left(P_{\varepsilon}\right)$ can be found as critical points of the functional $I_{\varepsilon}$ : $H^{1}(B) \rightarrow \mathbb{R}$ defined as

$$
I_{\varepsilon}(u)=I_{0}(u)-\varepsilon G(u)
$$

where the unperturbed functional $I_{0}^{c}(u)$ is defined by (see Sect. 2)

$$
I_{0}(u)=\frac{1}{2}\|u\|_{1}^{2}-\frac{1}{2^{*}} \int_{B}|u|^{2^{*}}-c(n-2) \int_{S^{n-1}}|u|^{2^{\frac{n-1}{n-2}}}
$$


and the perturbation $G$ has the form

$$
G(u)=\frac{1}{2^{*}} \int_{B} K(x)|u|^{2^{*}} d x+(n-2) \int_{S^{n-1}} h(x)|u|^{2 \frac{n-1}{n-2}} d \sigma .
$$

The existence of critical points of $I_{\varepsilon}$ will be faced by means of the perturbation theory studied in [1]. Precisely, let us recall that $I_{0}$ possesses an $n$-dimensional manifold $Z=Z^{c}$, given by (6). Moreover, $Z$ is non-degenerate in the sense that (i) - (ii) of Lemma 3 hold true. Then the results of [1] lead to consider the finite dimensional functional $\Gamma:=\left.G\right|_{Z}$ and give rise to the following Theorem:

Theorem 5. In the preceding setting, let us suppose that either

(a) $\Gamma$ has a strict maximum (minimum) on $Z$; or

(b) there exists an open subset $\Omega \subset \subset Z$ such that $\operatorname{deg}\left(\Gamma^{\prime}, \Omega, 0\right) \neq 0$.

Then $I_{\varepsilon}$ has a critical point close to $Z$, provided $\varepsilon$ is small enough.

In our specific case, the function $\Gamma(\mu, \xi)=G\left(z_{\mu, \xi}\right)$ has the expression

$$
\Gamma(\mu, \xi)=\frac{1}{2^{*}} \int_{B} K(x) z_{\mu, \xi}^{2^{*}}(x) d x+(n-2) \int_{S^{n-1}} h(\sigma) z_{\mu, \xi}^{\frac{(n-1)}{(n-2)}}(\sigma) d \sigma,
$$

where $\mu>0$ and $\xi \in \mathbb{R}^{n}$ are related to $c$ by (5), namely by

$$
\mu^{2}+|\xi|^{2}-c \kappa \mu-1=0 .
$$

In order to apply the preceding abstract result we need to study the behaviour of $\Gamma$ at the boundary of $Z$, which is given by

$$
\partial Z=\left\{z_{\mu, \xi_{0}}: \mu=0,\left|\xi_{0}\right|=1\right\} .
$$

The following lemma will be proved in the Appendix and describes the behaviour of $\Gamma$ at $\partial Z$. Below $a_{1}, \ldots, a_{6}$ denote positive constants defined in the Appendix.

Lemma 9. Let $\left|\xi_{0}\right|=1$ and let $v$ denote the outher normal direction to $\partial Z$ at $\left(0, \xi_{0}\right) . \Gamma$ can be extended to $\partial Z$ and there results:

(a) $\Gamma\left(0, \xi_{0}\right)=a_{1} K\left(\xi_{0}\right)+a_{2} h\left(\xi_{0}\right)$;

(b) $\partial_{v} \Gamma\left(0, \xi_{0}\right)=a_{3} K^{\prime}\left(\xi_{0}\right) \cdot \xi_{0}$;

(c) suppose that $K^{\prime}\left(\xi_{0}\right) \cdot \xi_{0}=0$ and let $n>3$. Then

$$
\partial_{\nu}^{2} \Gamma\left(0, \xi_{0}\right)=4\left[a_{4} \Delta_{T} K\left(\xi_{0}\right)+a_{5} D^{2} K\left(\xi_{0}\right)\left[\xi_{0}, \xi_{0}\right]+a_{6} \Delta_{T} h\left(\xi_{0}\right)\right] .
$$

Furthermore, if $n=3$ and $\Delta_{T} h\left(\xi_{0}\right) \neq 0$, then

$$
\partial_{\nu}^{2} \Gamma\left(0, \xi_{0}\right)= \begin{cases}+\infty & \text { provided } \Delta_{T} h\left(\xi_{0}\right)>0, \\ -\infty & \text { provided } \Delta_{T} h\left(\xi_{0}\right)<0 .\end{cases}
$$

The above Lemma is the counterpart of the calculation carried out in [2] for the Scalar Curvature Problem on $S^{n}$. 


\subsection{A general existence result}

Let us consider the auxiliary function $\psi: S^{n-1} \rightarrow \mathbb{R}$ defined by

$$
\psi(x)=a_{1} K(x)+a_{2} h(x), \quad x \in S^{n-1} .
$$

If $x \in \operatorname{Crit}(\psi)$ we denote by $m(x, \psi)$ its Morse index.

\section{Theorem 6. Suppose that either}

(a) there exists an absolute maximum

(resp. minimum) $p \in S^{n-1}$ of $\psi$ such that $K^{\prime}(p) \cdot p<0$ (resp.

$\left.K^{\prime}(p) \cdot p>0\right)$;

or

(b) $\psi$ is a Morse function satisfying

$$
\begin{gathered}
K^{\prime}(x) \cdot x \neq 0, \quad \forall x \in \operatorname{Crit}(\psi) ; \\
\sum_{x \in \operatorname{Crit}(\psi), K^{\prime}(x) \cdot x<0}(-1)^{m(x, \psi)} \neq 1 .
\end{gathered}
$$

Then for $|\varepsilon|$ sufficiently small, problem $\left(P_{\varepsilon}\right)$ has a positive solution.

Proof. We look for critical points of $\Gamma$ on $Z \simeq B$. Lemma 9-(a) and the notation introduced before says that $\left.\Gamma\right|_{\partial Z}=\psi$

(a) Let $p_{0}$ denote the point where $\Gamma$ achieves its absolute maximum on the compact set $\bar{Z}=Z \cup \partial Z$. Lemma 9-(b) and the preceding assumption (a)

imply that $p_{0} \in Z$. Then the existence of a critical point of $I_{\varepsilon}$, for $|\varepsilon|$ small, follows from Theorem 5-(a).

(b) According to Lemma 9-(b), if (39) holds then $\partial_{\nu} \Gamma(p) \neq 0$ at any critical point of $\left.\Gamma\right|_{\partial Z}$. Hence $\Gamma$ satisfies the general boundary conditions on $\partial Z$, see [19]. Moreover, setting

$$
\partial Z^{-}=\left\{\left(0, \xi_{0}\right) \in \partial Z: \partial_{\nu} \Gamma\left(\xi_{0}\right)<0\right\},
$$

there results

$$
\partial Z^{-}=\left\{\left(0, \xi_{0}\right):\left|\xi_{0}\right|=1, K^{\prime}\left(\xi_{0}\right) \cdot \xi_{0}<0\right\} .
$$

In particular, the critical points of $\psi$ on the negative boundary $\partial Z^{-}$are precisely the $x \in \operatorname{Crit}(\psi)$ such that $K^{\prime}(x) \cdot x<0$. Then, by a well known formula, see [13], we infer that

$$
\operatorname{deg}\left(\Gamma^{\prime}, Z, 0\right)=1-\sum_{x \in \operatorname{Crit}(\psi): K^{\prime}(x) \cdot x<0}(-1)^{m(x, \psi)} .
$$

Hence, by (40), $\operatorname{deg}\left(\Gamma^{\prime}, Z, 0\right) \neq 0$ and Theorem 5-(b) applies yielding the existence of a critical point of $I_{\varepsilon}$, for $|\varepsilon|$ small. 
Remark 5. (a) If $h \equiv 0$ then $\psi$ equals, up to the positive constant $a_{1}, K$. Hence the assumption made in case $(b)$ is precisely condition $\left(K_{1}\right)$, while (39) and (40), are nothing but conditions $\left(K_{2}\right)$ and $\left(K_{3}\right)$. As a consequence, Theorem 6-( $a$ ) implies Theorem 2 and Theorem 6- $(b)$ implies Theorem 3.

(b) Theorem 6-(b) is the counterpart of the results of [16] where it is taken $c=h=0$ but $R^{\prime}$ is possibly not close to a constant. Conditions like $(b)$ are reminiscent of conditions used by Bahri-Coron [8] dealing with the scalar curvature problem on $S^{3}$, see also $[2,17]$ for results on $S^{n}$. In contrast, assumption (a) is a new feature due to the presence of the boundary and has no counterpart in the problem on all $S^{n}$.

(c) Theorem 6 can be the starting point to prove a global result, see [18]. Here we limit ourselves to point out that (41) can be used to evaluate the degree of $I_{\varepsilon}^{\prime}$. Actually, since $z$ is a Mountain Pass critical point, the multiplicative property of the degree immediately implies that

$$
\operatorname{deg}\left(I_{\varepsilon}^{\prime}, B_{r}, 0\right)=(-1) \cdot \operatorname{deg}\left(\Gamma^{\prime}, Z, 0\right)=\sum_{x \in \operatorname{Crit}(\psi): K^{\prime}(x) \cdot x<0}(-1)^{m(x, \psi)}-1 .
$$

Our second general existence result deals with the case in which

$$
K^{\prime}(x) \cdot x=0, \quad \forall x \in \operatorname{Crit}(\psi) .
$$

In such a case, motivated by Lemma 9-(c), we introduce the function $\Psi: S^{n-1} \rightarrow$ $\mathbb{R}$,

$$
\Psi(x)=a_{4} \Delta_{T} K(x)+a_{5} D^{2} K(x)[x, x]+a_{6} \Delta_{T} h(x) .
$$

Let us note that, according to Lemma 9- $(c)$ there results $\partial_{v}^{2} \Gamma\left(0, \xi_{0}\right)=4 \Psi\left(\xi_{0}\right)$.

Theorem 7. Suppose that (43) holds and that

$$
\Psi(x) \neq 0, \quad \forall x \in \operatorname{Crit}(\psi) .
$$

Let $\psi$ be a Morse function and assume that

$$
\sum_{x \in \operatorname{Crit}(\psi), \Psi(x)<0}(-1)^{m(x, \psi)} \neq 1 .
$$

Furthermore, if $n=3$, we also assume that $\Delta_{T} h(x) \neq 0$ for all $x \in \operatorname{Crit}(\psi)$. Then for $|\varepsilon|$ sufficiently small, problem $\left(P_{\varepsilon}\right)$ has a solution

Proof. The proof will make use of arguments similar to those emploied for Theorem 6-(b). But, unlike above, the theory of critical points under general boundary conditions cannot be applied directly because now (43) implies that 
$\partial_{\nu} \Gamma=0$ at all the critical points of $\psi$. In order to overcome this problem, we consider for $\delta>0$ sufficiently small, the set $Z_{\delta}:=\{(\mu, \xi) \in Z: \mu>\delta\}$ with boundary $\partial Z_{\delta}=\{(\mu, \xi) \in Z: \mu=\delta\}$. Since $\psi$ is a Morse function, it readily follows that for any $\xi_{0} \in \operatorname{Crit}(\psi)$ there exists (for $\delta$ small enough) a unique $\xi_{\delta}$ such that

(i) $\quad\left(\delta, \xi_{\delta}\right) \in \partial Z_{\delta}$ and $\xi_{\delta} \rightarrow \xi_{0}$ as $\delta \rightarrow 0$;

(ii) $\xi_{\delta}$ is a critical point of $\left.\Gamma\right|_{\partial Z_{\delta}}$; moreover, $\left.\Gamma\right|_{\partial Z_{\delta}}$ has no other critical point but $\xi_{\delta}$;

(iii) the Morse index of $\xi_{\delta}$ is the same $m\left(\xi_{0}, \psi\right)$;

Furthermore, we claim that,

(iv) $\Gamma$ verifies the general boundary conditions on $Z_{\delta}$.

Actually, (44), or $\Delta_{T} h\left(\xi_{0}\right) \neq 0$ if $n=3$, jointly with Lemma 9-(c), implies that $\partial_{\nu} \Gamma\left(\delta, \xi_{\delta}\right) \neq 0$ for $\delta$ small. More precisely, $\partial_{\nu} \Gamma\left(\delta, \xi_{\delta}\right)<0$ iff $\xi_{\delta} \rightarrow \xi_{0}$ with $\Psi\left(\xi_{0}\right)<0$. Therefore, the critical points of $\left.\Gamma\right|_{\partial Z_{\delta}}$ on the negative boundary $\partial Z_{\delta}^{-}$ are in one-to-one correspondence with the $x \in \operatorname{Crit}(\psi)$ such that $\Psi(x)<0$. From the above arguments we infer that

$$
\operatorname{deg}\left(\Gamma^{\prime}, Z_{\delta}, 0\right)=1-\sum_{x \in \operatorname{Crit}(\psi): \Psi(x)<0}(-1)^{m(x, \psi)} .
$$

Then (45) implies that $\operatorname{deg}\left(\Gamma^{\prime}, Z_{\delta}, 0\right) \neq 0$ and the result follws.

Remark 6. (a) If $K \equiv 0$ then, up to positive constants, $\psi=h$ and $\Psi=\Delta_{T} h$ and thus Theorem 4 is a particular case of Theorem 7.

(b) It can be shown that our arguments can be adapted to handle an equation like (1) with $R^{\prime}=\varepsilon K$ and $h^{\prime}=c+\varepsilon h$, which can be seen as an extension of (3) where $R^{\prime}=0$ and $c=1$ is taken. This would lead to improve the results of [9]. For brevity, we do not carry out the details here.

(c) In all the above results we can deal with $-\Gamma$ instead of $\Gamma$. In such a case the condition (40) or (45) become $\sum_{x \in \operatorname{Crit}(\psi), \Psi(x)>0}(-1)^{m(x, \psi)} \neq(-1)^{n-1}$, $\sum_{x \in \operatorname{Crit}(\psi), K^{\prime}(x) \cdot x>0}(-1)^{m(x, \psi)} \neq(-1)^{n-1}$, respectively.

\subsection{The symmetric case}

When $K$ and $h$ inherit a symmetry one can obtain much more general results. They can be seen as the counterpart of the ones dealing with the Scalar Curvature problem on $S^{n}$ discussed in [4].

Theorem 8. Let us suppose that $K$ and $h$ are invariant under the action of a group of isometries $\Sigma \subset \mathbf{O}(n)$, such that $\operatorname{Fix}(\Sigma)=0 \in \mathbb{R}^{n}$. Then for $|\varepsilon|$ sufficiently small, problem $\left(P_{\varepsilon}\right)$ has a solution. 
Proof. The proof relies on the arguments of [4, Sec. 4]. For the sake of brevity, we will be sketchy, referring to such a paper for more details. We use the finite dimensional reduction discussed in the Sect. 3.2, with $I^{c}=I_{\varepsilon}$ and $Z^{c}=Z$, see Remark 1. From those results we infer that the manifold

$$
Z_{\varepsilon}=\left\{z_{\mu, \xi}+w_{\varepsilon}\left(z_{\mu, \xi}\right): \mu, \xi \text { satisfying (5) }\right\}
$$

is a natural constraint for $I_{\varepsilon}$. Let us recall that here $w=w_{\varepsilon}\left(z_{\mu, \xi}\right)$ is the solution of the equation

$$
\nabla I_{\varepsilon}\left(z_{\mu, \xi}+w\right) \in T_{z_{\mu, \xi}} Z
$$

According to Remark 1, it suffices to find a critical point of $\Phi_{\varepsilon}(\mu, \xi):=I_{\varepsilon}\left(z_{\mu, \xi}+\right.$ $\left.w_{\varepsilon}\left(z_{\mu, \xi}\right)\right)$. It is possible to show that $\Phi_{\varepsilon}$ is invariant under the action $\tau$ of a group acting on $Z$ and depending upon $\Sigma$. Moreover, from the fact that $\operatorname{Fix}(\Sigma)=\{0\}$ it follows that $(\mu, \xi) \in \operatorname{Fix}(\tau)$ iff $\xi=0$ and (hence) $\mu=$ $\mu_{0}:=\frac{1}{2}\left(c \kappa+\sqrt{c^{2} \kappa^{2}+4}\right)$. Plainly, $\Phi_{\varepsilon}$ has a critical point at $\mu=\mu_{0}, \xi=0$, which gives rise to a solution of $\left(P_{\varepsilon}\right)$.

For the reader convenience, let us give some more details in the specific case that $K$ and $h$ are even functions, when the arguments do not require new notation. We claim that if $K$ and $h$ are even then $\Phi_{\varepsilon}$ is invariant under the action $\tau$ given by $\tau:(\mu, \xi) \mapsto(\mu,-\xi)$. In other words, we will show that there results

$$
\Phi_{\varepsilon}(\mu, \xi)=\Phi_{\varepsilon}(\mu,-\xi) .
$$

In order to prove (46), we first remark that $z_{\mu,-\xi}(x)=z_{\mu, \xi}(-x)$. From this and using the fact that $K$ and $h$ are even, one checks that $w=w_{\varepsilon}\left(z_{\mu, \xi}\right)(-x)$ satisfies the equation, defining the natural constraint $Z_{\varepsilon}$,

$$
\nabla I_{\varepsilon}\left(z_{\mu,-\xi}+w\right) \in T_{z_{\mu,-\xi}} Z,
$$

By uniqueness, it follows that $w_{\varepsilon}\left(z_{\mu, \xi}\right)(-x)=w_{\varepsilon}\left(z_{\mu,-\xi}\right)(x)$. Then one infers:

$$
\begin{aligned}
I_{\varepsilon}\left(z_{\mu,-\xi}(x)+w_{\varepsilon}\left(z_{\mu,-\xi}\right)(x)\right) & =I_{\varepsilon}\left(z_{\mu, \xi}(-x)+w_{\varepsilon}\left(z_{\mu, \xi}\right)(-x)\right) \\
& =I_{\varepsilon}\left(z_{\mu, \xi}+w_{\varepsilon}\left(z_{\mu, \xi}\right)\right)
\end{aligned}
$$

proving (46).

Remark 7. (a) Coming back to the Scalar Curvature problem on the upper half sphere $S_{+}^{n}$, an even function $K$ corresponds to prescribing a scalar curvature on $S_{+}^{n}$ which is invariant under the symmetry $\left(x_{1}, \ldots, x_{n}, x_{n+1}\right) \mapsto$ $\left(-x_{1}, \ldots,-x_{n}, x_{n+1}\right)$.

(b) Using again the arguments of [4] one could treat the invariance under a group $\Sigma$ such that $\operatorname{Fix}(\Sigma) \neq\{0\}$. 


\section{A. Appendix}

\section{A.1. Proofs of technical Lemmas}

First we prove

Lemma 10. Given $M>0$, there exists $C>0$ such that for all $c>-M$ there holds

$$
\|z\| \leq C \cdot(1+|c|)^{-\frac{n-2}{2}} \quad \text { for all } z \in Z^{c} .
$$

Proof. By symmetry it suffices to take By symmetry it suffices to take $\xi=0$ and consider $z=z_{\mu}$. As $c \rightarrow+\infty$ one has that $\mu \sim \kappa c$ and $z_{\mu} \sim \mu^{(n-2) / 2}$ in $B$. Then the lemma follows by a straight calculation.

Now we start by proving Eq. (15). Since it is clearly $\nabla I_{0}^{c}(z)=0$, it is sufficient to estimate the quantity $\left\|\nabla I^{c}(z)-\nabla I_{0}^{c}(z)\right\|$. Given $v \in H^{1}(B)$ and setting

$$
\begin{aligned}
& \alpha_{1}=4 \frac{(n-1)}{(n-2)} \int_{B} \nabla_{g} z \cdot \nabla_{g} v d V_{g}-4 \frac{(n-1)}{(n-2)} \int_{B} \nabla z \cdot \nabla v d V_{0} ; \\
& \alpha_{2}=\int_{B} R_{g} z v d V_{g} ; \\
& \alpha_{3}=\int_{B} z^{\frac{n+2}{n-2}} v d V_{0}-\int_{B} z^{\frac{n+2}{n-2}} v d V_{g} ; \quad \alpha_{4}=2(n-1) \int_{\partial B} h_{g} z v d \sigma_{g} ; \\
& \alpha_{5}=2(n-1) c \int_{\partial B} z^{\frac{n}{n-2}} v d \sigma_{g}-2(n-1) c \int_{\partial B} z^{\frac{n}{n-2}} v d \sigma_{0},
\end{aligned}
$$

there holds

$$
\left(\nabla I^{c}(z)-\nabla I_{0}^{c}(z), v\right)=\alpha_{1}+\alpha_{2}+\alpha_{3}+\alpha_{4}+\alpha_{5} .
$$

As far as $\alpha_{1}$ is concerned, taking into account of equations (12), (14) and the fact that $\|z\| \leq C \cdot(1+|c|)^{-\frac{n-2}{2}}$ (see Lemma 10) one deduces that

$$
\begin{aligned}
\left|\alpha_{1}\right| & \leq C \int_{B}\left|\nabla_{g} z \cdot \nabla_{g} v-\nabla z \cdot \nabla v\right| d x+C \int_{B}|\nabla z \cdot \nabla v|\left|d V_{g}-d V_{0}\right| \\
& \leq C \cdot \varepsilon \cdot(1+|c|)^{-\frac{n-2}{2}} \cdot\|v\| .
\end{aligned}
$$

Turning to $\alpha_{2}$ we recall that the expression of $R_{g}$ as a function of $g$, is of the type

$$
R_{g}=D \Gamma+G^{2} ; \quad \Gamma=D g, \quad \Rightarrow \quad R_{g}=D^{2} g+(D g)^{2} .
$$

We start by estimating the quantity $\int_{B} R_{g} z v d V_{0}$. Integrating by parts, the term $\int_{B} D^{2} g z v d V_{0}$ transforms into

$$
\int_{B} D^{2} g z v d V_{0}=\int_{\partial B} D g z v d \sigma_{0}+\int_{B} D g D(z v) d V_{0} .
$$


Hence, if $g \in \mathcal{G}_{\varepsilon}$ (see expression (2)), from the Hölder inequality it follows that

$$
\int_{B} R_{g} z v d V_{0} \simeq \int_{B}\left(D^{2} g+(D g)^{2}\right) z v d V_{0} \leq C \cdot \varepsilon \cdot\|z\| \cdot\|v\|,
$$

and hence

$$
\left|\alpha_{2}\right| \leq \int_{B}\left|R_{g} z v\right| d V_{0}+\int_{B}\left|R_{g} z v\right|\left|d V_{g}-d V_{0}\right| \leq C \cdot \varepsilon \cdot(1+|c|)^{-\frac{n-2}{2}} \cdot\|v\| .
$$

With simple estimates one can also prove that

$$
\left|\alpha_{3}\right| \leq C \cdot \varepsilon \cdot(1+|c|)^{-\frac{n+2}{2}} \cdot\|v\| .
$$

The function $h_{g}$ is of the form $h_{g}=D g$ so, taking into account (2) one finds

$$
\left|\alpha_{4}\right| \leq C \cdot \varepsilon \cdot(1+|c|)^{-\frac{n-2}{2}} \cdot\|v\| .
$$

In order to estimate the last term $\alpha_{5}$, using the continuous embedding $H^{1}(B) \hookrightarrow$ $L^{2 \frac{n-1}{n-2}}\left(S^{n-1}\right)$ and the Hölder inequality one deduces that

$$
\left|\alpha_{5}\right| \leq C \cdot \varepsilon \cdot(1+|c|) \cdot\|z\|_{L^{\frac{n}{n-2}\left(S^{n-1}\right)}}^{\frac{n}{n-2}} \cdot\|v\| \leq C \cdot \varepsilon \cdot(1+|c|) \cdot(1+|c|)^{-\frac{n}{2}} \cdot\|v\| .
$$

Putting together equations (49)-(52) one deduces (15).

Turning to equation (19) and given $v_{1}, v_{2} \in H^{1}(B)$, there holds

$$
\left(D^{2} I^{c}(z+w)-D^{2} I^{c}(z)\right)\left[v_{1}, v_{2}\right]=\delta_{1}+\delta_{2},
$$

where

$$
\begin{aligned}
& \delta_{1}=\frac{(n+2)}{(n-2)}\left(\int_{B} u^{\frac{4}{n-2}} v_{1} v_{2} d V_{g}-\int_{B}(u+w)^{\frac{4}{n-2}} v_{1} v_{2} d V_{g}\right) \\
& \delta_{2}=2 n \frac{(n-1)}{(n-2)} c\left(\int_{\partial B} u^{\frac{2}{n-2}} v_{1} v_{2} d \sigma_{g}-\int_{\partial B}(u+w)^{\frac{2}{n-2}} v_{1} v_{2} d \sigma_{g}\right) .
\end{aligned}
$$

Using standard inequalities one finds that

$$
\begin{gathered}
\left|\delta_{1}\right| \leq \begin{cases}C \cdot\|w\|^{\frac{4}{n-2}} & \text { for } n \geq 6, \\
C \cdot\|w\| \cdot\left(\|u\|^{\frac{6-n}{n-2}}+\|w\|^{\frac{6-n}{n-2}}\right) & \text { for } n<6 ;\end{cases} \\
\left|\delta_{2}\right| \leq \begin{cases}C \cdot(1+|c|) \cdot\|w\|^{\frac{4}{n-2}} & \text { for } n \geq 4, \\
C \cdot(1+|c|) \cdot\|w\| \cdot\left(\|u\|^{\frac{4-n}{n-2}}+\|w\|^{\frac{4-n}{n-2}}\right) & \text { for } n<4,\end{cases} \\
\left\|D^{2} I^{c}(z+w)-D^{2} I^{c}(z)\right\| \leq C \cdot|c| \cdot\|w\|^{\frac{2}{n-2}} .
\end{gathered}
$$


so we obtain the estimate.

We now prove inequality (16). Given $v_{1}, v_{2} \in H^{1}(B)$ and setting

$$
\begin{aligned}
& \beta_{1}=4 \frac{(n-1)}{(n-2)} \int_{B} \nabla_{g} v_{1} \cdot \nabla_{g} v_{2} d V_{g}-4 \frac{(n-1)}{(n-2)} \int_{B} \nabla v_{1} \cdot \nabla v_{2} d V_{0} ; \\
& \beta_{2}=\int_{B} R_{g} v_{1} v_{2} d V_{g} ; \\
& \beta_{3}=\frac{(n+2)}{(n-2)} \int_{B} z^{\frac{4}{n-2}} v_{1} v_{2} d V_{0}-\frac{(n+2)}{(n-2)} \int_{B} z^{\frac{4}{n-2}} v_{1} v_{2} d V_{g} ; \\
& \beta_{4}=2(n-1) \int_{\partial B} h_{g} v_{1} v_{2} d \sigma_{g} ; \\
& \beta_{5}=2 n \frac{(n-1)}{(n-2)} c \int_{\partial B} z^{\frac{2}{n-2}} v_{1} v_{2} d \sigma_{g}-2 n \frac{(n-1)}{(n-2)} c \int_{\partial B} z^{\frac{2}{n-2}} v_{1} v_{2} d \sigma_{0},
\end{aligned}
$$

there holds

$$
\left(D^{2} I^{c}(z)-D^{2} I_{0}^{c}(z)\right)\left[v_{1}, v_{2}\right]=\beta_{1}+\beta_{2}+\beta_{3}+\beta_{4}+\beta_{5} .
$$

For $\beta_{1}$, taking into account equation (14) one finds

$$
\begin{aligned}
\left|\beta_{1}\right| & \leq C \int_{B}\left|\nabla_{g} v_{1} \cdot \nabla_{g} v_{2}-\nabla v_{1} \cdot \nabla v_{2}\right| d V_{0}+C \int_{B}\left|\nabla v_{1} \cdot \nabla v_{2}\right| \cdot\left|d V_{g}-d V_{0}\right| \\
& \leq C \cdot \varepsilon \cdot\left\|v_{1}\right\| \cdot\left\|v_{2}\right\| .
\end{aligned}
$$

Turning to $\beta_{2}$ reasoning as for the above term $\alpha_{2}$ one deduces that

$$
\left|\beta_{2}\right| \leq \int_{B}\left|R_{g} z v\right| d V_{g} \leq C \cdot \varepsilon \cdot\left\|v_{1}\right\| \cdot\left\|v_{2}\right\| .
$$

In the same way one can prove that

$$
\left|\beta_{3}\right| \leq C \cdot \varepsilon \cdot\|z\|^{\frac{4}{n-2}} \cdot\left\|v_{1}\right\| \cdot\left\|v_{2}\right\| \leq C \cdot \varepsilon \cdot(1+|c|)^{-2} \cdot\left\|v_{1}\right\| \cdot\left\|v_{2}\right\| .
$$

For the term $\beta_{4}$, similarly to the expression $\alpha_{4}$ above there holds

$$
\left|\beta_{4}\right| \leq C \cdot \varepsilon \cdot\left\|v_{1}\right\| \cdot\left\|v_{2}\right\| .
$$

Turning to $\beta_{5}$, using the Hölder inequality one deduces that

$$
\left|\beta_{5}\right| \leq C \cdot c \cdot \varepsilon \cdot(1+|c|) \cdot\|z\|_{L^{\frac{2}{n-2}\left(S^{n-1}\right)}}^{\frac{2}{n-2}} \cdot\left\|v_{1}\right\| \cdot\left\|v_{2}\right\| \leq C \cdot \varepsilon \cdot\left\|v_{1}\right\| \cdot\left\|v_{2}\right\| .
$$

Putting together equations (55)-(59) (59) one deduces inequality (16).

Equation (17) follows from similar computations. 


\section{A.2. Proof of Lemma 9}

Given $\xi_{0} \mid=1$, we introduce a reference frame in $\mathbb{R}^{n}$ such that $e_{n}=-\xi_{0}$. Let $\alpha=\alpha(\mu)$ be such that the pair $(\mu, \xi)$, with $\xi=\alpha \xi_{0}$, satisfies (5). Setting

$$
\gamma(\mu)=\Gamma\left(\mu,-\alpha(\mu) e_{n}\right),
$$

one has that

$$
\Gamma\left(0, \xi_{0}\right)=\gamma(0), \quad \partial_{\nu} \Gamma\left(0, \xi_{0}\right)=-\gamma^{\prime}(0), \quad \partial_{\nu}^{2} \Gamma\left(0, \xi_{0}\right)=\gamma^{\prime \prime}(0) .
$$

In order to evaluate the above quantities, it is convenient to make a change of variables. This will considerably simplify the calculation when we deal with $\gamma^{\prime}(0)$ and $\gamma^{\prime \prime}(0)$.

Let $\psi: \mathbb{R}_{+}^{n} \rightarrow B$ be the map given by

$$
\begin{aligned}
& \left(y^{\prime}, y_{n}\right) \in \mathbb{R}_{+}^{n} \rightarrow\left(x^{\prime}, x_{n}\right) \in B ; \\
& x^{\prime}=\frac{2 y^{\prime}}{\left(y^{\prime}\right)^{2}+\left(y_{n}+1\right)^{2}}, \quad x_{n}=\frac{\left(y^{\prime}\right)^{2}+y_{n}^{2}-1}{\left(y^{\prime}\right)^{2}+\left(y_{n}+1\right)^{2}} .
\end{aligned}
$$

Here and in the sequel,if $x \in \mathbb{R}^{n}$ we will set $x^{\prime}=\left(x_{1}, \ldots, x_{n-1}\right)$ so that $x=$ $\left(x^{\prime}, x_{n}\right)$.

By using simple computations it turns out that

$$
\gamma(\mu)=\tilde{\gamma}(\tilde{\mu}),
$$

where

$$
\tilde{\gamma}(\tilde{\mu})=\frac{1}{2^{*}} \int_{\mathbb{R}_{+}^{n}} \tilde{K}(y)\left(z_{\tilde{\mu}, 0}^{c}\right)^{2^{*}}(y) d y+(n-2) \int_{\partial \mathbb{R}_{+}^{n}} \tilde{h}(\omega)\left(z_{\tilde{\mu}, 0}^{c}\right)^{2 \frac{n-1}{n-2}}(\omega) d \omega,
$$

and

$$
\tilde{\mu}=\frac{2 \mu}{1+\mu^{2}+\alpha(\mu)} ; \quad \tilde{K}(y)=K(\psi(y)) .
$$

Let us point out that the derivatives of $K$ and $\tilde{K}$ satisfy the following relations:

$$
\begin{aligned}
D_{y_{n}} \tilde{K}(0,0) & =2 D_{x_{n}} K\left(\xi_{0}\right) \\
D_{y^{\prime}} \tilde{K}(0,0) & =2 D_{x^{\prime}}\left(\xi_{0}\right) ; \\
D_{y_{n}}^{2} \tilde{K}(0,0) & =4\left(D_{x_{n}}^{2} K-D_{x_{n}} K\right)\left(\xi_{0}\right) ; \\
D_{y^{\prime}}^{2} \tilde{K}(0,0) & =4\left(D_{x^{\prime}}^{2} K-D_{x_{n}} K\right)\left(\xi_{0}\right) ; \\
D_{y^{\prime}, y_{n}}^{2} \tilde{K}(0,0) & =4\left(D_{x^{\prime}, x_{n}}^{2} K-D_{x^{\prime}} K\right)\left(\xi_{0}\right) .
\end{aligned}
$$

The change of variables $y=\tilde{\mu} q, \omega=\tilde{\mu} \sigma$ yields

$$
\tilde{\gamma}(\tilde{\mu})=\frac{1}{2^{*}} \int_{\mathbb{R}_{+}^{n}} \tilde{K}(\tilde{\mu} q)\left(z_{1,0}^{c}\right)^{2^{*}}(q) d q+(n-2) \int_{\partial \mathbb{R}_{+}^{n}} \tilde{h}(\tilde{\mu} \sigma)\left(z_{1,0}^{c}\right)^{2 \frac{n-1}{n-2}}(\sigma) d \sigma .
$$


Hence, passing to the limit for $\tilde{\mu} \rightarrow 0$, it follows that

$$
\gamma(0)=\tilde{\gamma}(0)=a_{1} \tilde{K}(0)+a_{2} \tilde{h}(0)=a_{1} K\left(\xi_{0}\right)+a_{2} h\left(\xi_{0}\right),
$$

with

$$
a_{1}=\frac{1}{2^{*}} \int_{\mathbb{R}_{+}^{n}} z_{0}^{2^{*}}\left(q^{\prime}, q_{n}-\kappa c / 2\right) d q, \quad a_{2}=(n-2) \int_{\partial \mathbb{R}_{+}^{n}} z_{0}^{2 \frac{n-1}{n-2}}(\sigma, \kappa c / 2) d \sigma .
$$

Let us now evaluate the first derivative. There holds

$$
\gamma^{\prime}(0)=\frac{d \tilde{\gamma}}{d \tilde{\mu}}(0) \cdot \frac{d \tilde{\mu}}{d \mu}(0)=2 \tilde{\gamma}^{\prime}(0) .
$$

Moreover from formula (60) we deduce

$$
\begin{aligned}
\tilde{\gamma}^{\prime}(\tilde{\mu}) & =\frac{1}{2^{*}} \int_{\mathbb{R}_{n}^{+}} \nabla \tilde{K}(\tilde{\mu} q) \cdot q\left|z_{1,0}^{c}(q)\right|^{2^{*}} d q+(n-2) \\
& \times \int_{\partial \mathbb{R}_{+}^{n}} \nabla \tilde{h}(\tilde{\mu} \sigma) \cdot \sigma\left|z_{1,0}^{c}(\sigma)\right|^{2 \frac{n-1}{n-2}}(\sigma) d \sigma .
\end{aligned}
$$

For symmetry reasons when $\tilde{\mu} \rightarrow 0$, the parallel component to $\partial \mathbb{R}_{+}^{n}$ in the first integral and the second integral vanishes, hence it follows that

$$
\gamma^{\prime}(0)=2 \tilde{\gamma}^{\prime}(0)=\frac{2}{2^{*}} D_{n} \tilde{K}(0) \int_{\mathbb{R}_{+}^{n}} q_{n}\left|z_{1,0}^{c}(q)\right|^{2^{*}} d q=-a_{3} K^{\prime}\left(\xi_{0}\right) \cdot \xi_{0},
$$

where

$$
a_{3}=\frac{4}{2^{*}} \int_{\mathbb{R}_{+}^{n}} q_{n} z_{0}^{2^{*}}\left(q^{\prime}, q_{n}-\kappa c / 2\right) d q .
$$

We are interested in the study of the second derivative only in the case in which the first derivative vanishes, namely when $K^{\prime}\left(\xi_{0}\right) \cdot \xi_{0}=0$.

As for the second derivative, there holds:

$$
\begin{aligned}
\tilde{\gamma}^{\prime \prime}(\tilde{\mu})= & \frac{1}{2^{*}} \int_{\mathbb{R}_{n}^{+}} \sum_{i, j=1}^{n} D_{i j}^{2} \tilde{K}(\tilde{\mu} q) q_{i} q_{j}\left|z_{1,0}^{c}(q)\right|^{2^{*}} d q \\
& +(n-2) \int_{\partial \mathbb{R}_{+}^{n}} \sum_{i, j=1}^{n-1} D_{i j}^{2} \tilde{h}(\tilde{\mu} \sigma) \sigma_{i} \sigma_{j}\left|z_{1,0}^{c}(\sigma)\right|^{2 \frac{(n-1)}{(n-2)}} d \sigma \\
:= & \delta(\tilde{\mu})+\rho(\tilde{\mu}) .
\end{aligned}
$$

Now we have to distinguish the case $n=3$ and the case $n>3$. In fact the boundary integral $\rho(\tilde{\mu})$ in (63) is uniformly dominated by a function in $L^{1}\left(\partial \mathbb{R}_{+}^{n}\right)$ 
if and only if $n>3$. However it is possible to determine the sign of this integral also for $n=3$ : it turns out that

$$
\begin{aligned}
\lim _{\tilde{\mu} \rightarrow 0} \delta(\tilde{\mu})= & \frac{1}{2^{*}(n-1)} \int_{\mathbb{R}_{+}^{n}}\left|q^{\prime}\right|^{2}\left|z_{1,0}^{c}(q)\right|^{2^{*}} d q \cdot \Delta_{T} \tilde{K}(0) \\
& +\frac{1}{2^{*}} \int_{\mathbb{R}_{+}^{n}} q_{n}^{2}\left|z_{1,0}^{c}(q)\right|^{2^{*}} d q \cdot D_{n n}^{2} \tilde{K}(0) ;
\end{aligned}
$$

and

$$
\begin{cases}\lim _{\tilde{\mu} \rightarrow 0} \rho(\tilde{\mu} u)=(+\infty) \cdot \Delta_{T} \tilde{h}(0), & \text { for } n=3 ; \\ \lim _{\tilde{\mu} \rightarrow 0} \rho(\tilde{\mu})=\frac{(n-2)}{(n-1)} \int_{\partial \mathbb{R}_{+}^{n}}|\sigma|^{2}\left|z_{1,0}^{c}(\sigma)\right|^{2 \frac{(n-1)}{(n-2)}} d \sigma \cdot \Delta_{T} \tilde{h}(0), & \text { for } n>3 .\end{cases}
$$

Hence we have that

$$
\tilde{\gamma}^{\prime \prime}(0)= \begin{cases}(+\infty) \cdot \Delta_{T} h\left(\xi_{0}\right) & \text { for } n=3 \\ a_{4} \Delta_{T} K\left(\xi_{0}\right)+a_{5} D^{2} K\left(\xi_{0}\right)\left[\xi_{0}, \xi_{0}\right]+a_{6} \Delta_{T} h\left(\xi_{0}\right) & \text { for } n>3,\end{cases}
$$

where

$$
\begin{aligned}
& a_{4}=\frac{4}{(n-1) 2^{*}} \int_{\mathbb{R}_{+}^{n}}\left|q^{\prime}\right|^{2} z_{0}^{2^{*}}\left(q^{\prime}, q_{n}-\kappa c / 2\right) d q, \\
& a_{5}=\frac{4}{2^{*}} \int_{\mathbb{R}_{+}^{n}} q_{n}^{2} z_{0}^{2^{*}}\left(q^{\prime}, q_{n}-\kappa c / 2\right) d q, \\
& a_{6}=4 \frac{(n-2)}{(n-1)} \int_{\partial \mathbb{R}_{+}^{n}}|\sigma|^{2} z_{0}^{2 \frac{n-1}{n-2}}(\sigma, \kappa c / 2) d \sigma .
\end{aligned}
$$

Finally, since $\gamma^{\prime \prime}(0)=4 \tilde{\gamma}^{\prime \prime}(0)$, the lemma follows.

\section{References}

1. A. Ambrosetti, M. Badiale, Homoclinics: Poincaré-Melnikov type results via a variational approach, Ann. Inst. Henri. Poincaré Analyse Non Linéaire 15 (1998), 233-252. 323,

2. A. Ambrosetti, J. Garcia Azorero, I. Peral, Perturbation of $-\Delta u+u^{\frac{(N+2)}{(N-2)}}=0$, the Scalar Curvature Problem in $\mathbb{R}^{N}$ and related topics, J. Funct. Analysis, 165 (1999), 117-149.

3. A.Ambrosetti, A. Malchiodi, A multiplicity result for the Yamabe problem on $S^{n}$, J. Funct. Analysis, 168 (1999), 529-561.

4. Ambrosetti A., Malchiodi A., On the Symmetric Scalar Curvature Problem on $S^{n}$, J. Diff. Equations, 170-1 (2001), 228-245.

5. A.Ambrosetti, A. Malchiodi, Yan Yan Li, Scalar Curvature under Boundary Conditions, C. R. Acad. Sci. Paris, 330 (2000), 1013-1018.

6. T. Aubin, Some Nonlinear Problems in Differential Geometry, Springer, (1998). 
7. M.Berti, A. Malchiodi, Multiplicity results and multibump solutions for the Yamabe problem on $S^{n}$, J. Funct. Anal. 180 (2001), 210-241.

8. A.Bahri, J. M. Coron, The Scalar-Curvature problem on the standard three-dimensional sphere, J. Funct. Anal. 95 (1991), 106-172.

9. A. Chang, X. Xu, P. Yang, A perturbation result for prescribing mean curvature, Math. Annalen 310 (1998), 473-496.

10. P. Cherrier, Problemes de Neumann nonlinearires sur les varietes Riemanniennes, J. Funct. Anal. 57 (1984), 154-207.

11. J. Escobar, Conformal deformation of a Riemannian metric to a scalar fla metric with constant mean curvature on the boundary, Ann. Math. 136 (1992), 1-50.

12. J. Escobar, Conformal metrics with prescribed mean curvature on the boundary, Cal. Var. 4 (1996), 559-592.

13. D.H. Gottlieb, A De Moivre like formula for fixed point theory, Contemp. Math. 72 (1988), 99-105.

14. Z.C.Han, Yan Yan Li, The Yamabe problem on manifolds with boundaries: existence and compactness results, Duke Math. J. 99 (1999), 489-542.

15. Z.C. Han, Yan Yan Li, The existence of conformal metrics with constant scalar curvature and constant boundary mean curvature, Comm. Anal. Geom., to appear.

16. Yan Yan Li, The Nirenberg problem in a domain with boundary, Top. Meth. Nonlin. Anal. 6 (1995), 309-329.

17. Yan Yan Li, Prescribing scalar curvature on $S^{n}$ and related topics, Part I, J. Diff. Equat. 120 (1995), 319-410; Part II, Existence and compactness, Comm. Pure Appl. Math. 49 (1996), 437-477.

18. Z. Djadli, A. Malchiodi, M.O. Ahmedou, Prescribing scalar and boundary mean curvature on the half three sphere, preprint.

19. M. Morse, G. Van Schaak, The critical point theory under general boundary conditions, Annals of Math. 35 (1934), 545-571. 